\title{
STOCHASTIC LATTICE DYNAMICAL SYSTEMS WITH FRACTIONAL NOISE*
}

\author{
HAKIMA BESSAIH ${ }^{\dagger}$, MARÍA J. GARRIDO-ATIENZA ${ }^{\ddagger}$, XIAOYING HAN ${ }^{\S}$, AND \\ BJÖRN SCHMALFUSS
}

\begin{abstract}
This article studies stochastic lattice dynamical systems driven by a fractional Brownian motion with Hurst parameter $H \in(1 / 2,1)$. First of all, we investigate the existence and uniqueness of pathwise mild solutions to such systems by the Young integration setting and prove that the solution generates a random dynamical system. Further, we analyze the exponential stability of the trivial solution.
\end{abstract}

Key words. stochastic lattice equations, Hilbert-valued fractional Brownian motion, pathwise solutions, exponential stability

AMS subject classifications. Primary, 60H15; Secondary, 37L55, 60G22, 37K45

DOI. $10.1137 / 16 \mathrm{M} 1085504$

1. Introduction. Lattice dynamical systems arise in a wide range of applications where the spatial structure has a discrete character, such as image processing $[14,15,16,37]$, pattern recognition [12, 13], and chemical reaction theory [18, 29, 33]. In particular, lattice systems have been used in biological systems to describe the dynamics of pulses in myelinated axons where the membrane is excitable only at spatially discrete sites $[5,6,30,31,38,40]$. Lattice systems have also been used in fluid dynamics to describe the fluid turbulence in shell models (see, e.g., [7, 41]). For some cases, lattice dynamical systems arise as discretization of partial differential equations, while they can be interpreted as ordinary differential equations in Banach spaces which are often simpler to analyze.

Random effects arise naturally in these models to take into account the uncertainty (see, e.g., [26]). In this paper, we will consider the following stochastic lattice dynamical system (SLDS) with a diffusive adjacent neighborhood interaction, a dissipative nonlinear reaction term, and a fractional Brownian motion (fBm) at each node:

$$
d u_{i}(t)=\left(\nu\left(u_{i-1}-2 u_{i}+u_{i+1}\right)-\lambda u_{i}+f_{i}\left(u_{i}\right)\right) d t+\sigma_{i} h_{i}\left(u_{i}\right) d B_{i}^{H}(t), i \in Z,
$$

with initial condition $u_{i}(0)$, where $Z$ denotes the integers set, $\nu$ and $\lambda$ are positive constants, $u_{i}, \sigma_{i} \in R$, each $B_{i}^{H}(t)$ is a one-dimensional two-sided fBm with Hurst parameter $H \in(1 / 2,1)$, and $f_{i}$ and $h_{i}$ are smooth functions satisfying proper conditions.

\footnotetext{
${ }^{*}$ Received by the editors August 1, 2016; accepted for publication (in revised form) December 19, 2016; published electronically April 25, 2017.

http://www.siam.org/journals/sima/49-2/M108550.html

Funding: The work of the first author was supported by NSF grant DMS-1418838. The work of the second, third, and fourth authors was partially supported by European Funds MTM2015-63723-P (MINECO/FEDER, EU).

${ }^{\dagger}$ University of Wyoming, Laramie, WY 82071-3036 (bessaih@uwyo.edu).

${ }_{\ddagger}$ Departamento Ecuaciones Diferenciales y Análisis Numérico, Universidad de Sevilla, 41080-Sevilla, Spain (mgarrido@us.es).

$\S$ Department of Mathematics \& Statistics, Auburn University, Auburn, AL 36849 (xzh0003@ auburn.edu).

؟Institut für Mathematik, 07737, Jena, Germany (bjoern.schmalfuss@uni-jena.de).
} 
On the other hand, the theory of random dynamical systems (RDSs) has been developed by Arnold (see the monograph [1]) and his collaborators. Thanks to this theory, we can study the stability behavior of solutions of differential equations containing a general type of noise, in terms of random attractors and their dimensions, random fixed points, random inertial, stable or unstable manifolds, and Lyapunov exponents. Finite dimensional Itô equations with sufficiently smooth coefficients generate RDSs. This assertion follows from the flow property generated by the Itô equation, due to Kolmogorov's theorem for a Hölder-continuous version of a random field with finitely many parameters; see [32]. However this method fails for infinite dimensional stochastic equations, i.e., for systems with infinitely many parameters, and in particular for SLDSs. To justify the flow property or the generation of an RDS by an SLDS, a special transform technique has been used in the literature. Such a transform reformulates an SLDS to a pathwise random differential equation, by using Ornstein-Uhlenbeck processes. But this technique applies only to SLDSs with random perturbations given by either an additive white noise $\sigma_{i} d B_{i}^{1 / 2}(t)$ or a simple multiplicative white noise $\sigma_{i} u_{i} d B_{i}^{1 / 2}(t)$ at each node $i \in Z$ (see [2, 3, 8, 9, 27, 28] and the references therein). Nevertheless, there are some recent works where the generation of an RDS is established for the solution of abstract stochastic differential equations and stochastic evolution equations without transformation into random systems; see $[21,22,25]$, where $H \in(1 / 2,1)$, and [23, 24], where $H \in(1 / 3,1 / 2]$. Note that in these last two papers the case of a Brownian motion $B^{1 / 2}$ is considered, giving a positive answer to the rather open problem of the generation of RDSs for systems with general diffusion noise terms.

Our main goal in this paper is to develop new techniques of stochastic analysis to analyze the dynamics of SLDSs perturbed by general fBms with Hurst parameter $H \in(1 / 2,1)$. In probability theory, an $\mathrm{fBm}$ is a centered Gauss process with a special covariance function determined by the Hurst parameter $H \in(0,1)$. For $H=1 / 2$, $B^{1 / 2}$ is the Brownian motion where the generalized temporal derivative is the white noise. For $H \neq 1 / 2, B^{H}$ is not a semimartingale and, as a consequence, classical techniques of stochastic analysis are not applicable. In particular, the fBm with a Hurst parameter $H \in(1 / 2,1)$ enjoys the property of a long-range memory, which roughly implies that the decay of stochastic dependence with respect to the past is only subexponentially slow. This long-range dependence property of the fBm makes it a realistic choice of noise for problems with long memory in the applied sciences.

In this paper, we prove the existence of a unique mild solution for system (1.1) and analyze the exponential stability of the trivial solution. The existence of the unique solution for a fixed initial condition relies on a fixed point argument, based on nice estimates satisfied by the stochastic integral with an fBm as integrator. Further, we prove that the trivial solution of the SLDS is exponentially stable, namely, assuming that zero is a solution of the SLDS, then any other solution converges to the trivial solution exponentially fast, provided that the corresponding initial data belongs to a random neighborhood of zero. Since we do not transform the underlying SLDS into a random equation, the norm of any nontrivial solution depends on the magnitude of the norm of the noisy input. Therefore to obtain stability we develop a cut-off argument, by which the functions appearing in the SLDS only need to be defined in a small time interval $[-\delta, \delta]$. This brings up the idea of considering the composition of the functions defined locally with a cut-off-like function depending on a random variable $\hat{R}$. With these compositions, we construct a sequence $\left(u^{n}\right)_{n \in N}$ such that each element $u^{n}$ is a solution of a modified SLDS on $[0,1]$ driven by a path of the 
fBm depending also on $n$. It is easily conceived that we will require $u^{n}(0)=u^{n-1}(1)$. The norm of each $u^{n}$ depends on the magnitude of the corresponding driving noise and a new random variable $R$ related to the aforementioned $\hat{R}$. By a suitable choice of these random variables, we can apply a discrete Gronwall-like lemma to obtain a subexponential estimate of every element of the sequence. Finally, temperedness comes into play in order to ensure that $\left(u^{n}\right)_{n \in N}$ describes the solution of our SLDS on the positive real line, and such a solution converges to the equilibrium given by the trivial solution exponentially fast.

Recently, in [20] the authors have considered a stochastic differential equation perturbed by a Hölder-continuous function with Hölder exponent greater than $1 / 2$ and have investigated the exponential stability of the trivial solution. In this paper we extend the study of the longtime stability with exponential decay to the case of considering infinite dimensional dynamical systems. We also would like to announce the forthcoming paper [19], where the authors show that the trivial solution is globally attractive, by using a technique based on a suitable choice of stopping times that depend on the noise signal, and that shall play the key role in establishing the stability results.

The rest of this paper is organized as follows. In section 2 we provide necessary preliminaries and some prior estimates to be used in the paper, in section 3 we study the existence and uniqueness of pathwise solutions to (1.1), and in section 4 we investigate the stability of solutions to our SLDS. Section 5, the appendix, introduces some lemmas that are used in section 4.

2. Preliminaries. Denote by

$$
\ell^{2}:=\left\{\left(u_{i}\right)_{i \in Z}: \sum_{i \in Z} u_{i}^{2}<\infty\right\}
$$

the separable Hilbert space of square summable sequences, equipped with the norm

$$
\|u\|:=\left(\sum_{i \in Z} u_{i}^{2}\right)^{\frac{1}{2}}, \quad u=\left(u_{i}\right)_{i \in Z} \in \ell^{2},
$$

and the inner product

$$
\langle u, v\rangle=\sum_{i \in Z} u_{i} v_{i}, \quad u=\left(u_{i}\right)_{i \in Z}, v=\left(v_{i}\right)_{i \in Z} \in \ell^{2} .
$$

Let us consider the infinite sequence $\left(\mathbf{e}_{i}\right)_{i \in Z}$, where $\mathbf{e}_{i}$ denotes the element in $\ell^{2}$ having 1 at position $i$ and 0 elsewhere. Then $\left(\mathbf{e}_{i}\right)_{i \in Z}$ forms a complete orthonormal basis of $\ell^{2}$.

Consider given $T_{1}<T_{2}$. Let $C^{\beta}\left(\left[T_{1}, T_{2}\right] ; \ell^{2}\right)$ be the Banach space of Höldercontinuous functions with exponent $0<\beta<1$ having values in $\ell^{2}$, with norm

$$
\|u\|_{\beta, \rho, T_{1}, T_{2}}=\|u\|_{\infty, \rho, T_{1}, T_{2}}+\|u\|_{\beta, \rho, T_{1}, T_{2}},
$$

where $\rho \geq 0$ and

$$
\begin{aligned}
\|u\|_{\infty, \rho, T_{1}, T_{2}} & =\sup _{s \in\left[T_{1}, T_{2}\right]} e^{-\rho\left(s-T_{1}\right)}\|u(s)\|, \\
\|u\|_{\beta, \rho, T_{1}, T_{2}} & =\sup _{T_{1} \leq s<t \leq T_{2}} e^{-\rho\left(t-T_{1}\right)} \frac{\|u(t)-u(s)\|}{(t-s)^{\beta}} .
\end{aligned}
$$

Copyright $\odot$ by SIAM. Unauthorized reproduction of this article is prohibited. 
For $\rho>0$ and $\rho=0$ the corresponding norms are equivalent. We will suppress the index $\rho$ in these notations if $\rho=0$, and we will suppress $T_{1}, T_{2}$ when $T_{1}=0$ and $T_{2}=1$.

Since confusion is not possible, later we will use the notation $\|\cdot\|_{\beta, \rho, T_{1}, T_{2}}$ to express the norms of $C^{\beta}\left(\left[T_{1}, T_{2}\right] ; R\right)$ and of $C^{\beta}\left(\left[T_{1}, T_{2}\right] ; L_{2}\left(\ell^{2}\right)\right)$, as well.

In order to define integrals with Hölder-continuous integrators, we next define Weyl fractional derivatives of functions on separable Hilbert spaces; see [39].

Definition 2.1. Let $V_{1}$ and $V_{2}$ be separable Hilbert spaces and let $0<\alpha<1$. The Weyl fractional derivatives of general measurable functions $Z:[s, t] \rightarrow V_{1}$ and $\omega:[s, t] \rightarrow V_{2}$, of order $\alpha$ and $1-\alpha$, respectively, are defined for $s<r<t$ by

$$
\begin{aligned}
D_{s+}^{\alpha} Z[r] & =\frac{1}{\Gamma(1-\alpha)}\left(\frac{Z(r)}{(r-s)^{\alpha}}+\alpha \int_{s}^{r} \frac{Z(r)-Z(q)}{(r-q)^{1+\alpha}} d q\right) \in V_{1}, \\
D_{t-}^{1-\alpha} \omega_{t-}[r] & =\frac{(-1)^{\alpha}}{\Gamma(\alpha)}\left(\frac{\omega(r)-\omega(t-)}{(t-r)^{1-\alpha}}+(1-\alpha) \int_{r}^{t} \frac{\omega(r)-\omega(q)}{(q-r)^{2-\alpha}} d q\right) \in V_{2},
\end{aligned}
$$

where

$$
\omega_{t-}(r)=\omega(r)-\omega(t-),
$$

and $\omega(t-)$ is the left side limit of $\omega$ at $t$.

The next result shows that Weyl fractional derivatives are well-posed for Höldercontinuous functions with suitable Hölder exponents. The proof follows easily and therefore we omit it.

Lemma 2.2. Suppose that $Z \in C^{\beta}\left(\left[T_{1}, T_{2}\right] ; V_{1}\right), \omega \in C^{\beta^{\prime}}\left(\left[T_{1}, T_{2}\right] ; V_{2}\right), T_{1} \leq s<$ $t \leq T_{2}$ and that $1-\beta^{\prime}<\alpha<\beta$. Then $D_{s+}^{\alpha} Z$ and $D_{t-}^{1-\alpha} \omega_{t-}$ are well-defined.

Let us assume for a while that $V_{1}=V_{2}=R$. Following Zähle [42] we can define the fractional integral by

$$
\int_{s}^{t} Z d \omega=(-1)^{\alpha} \int_{s}^{t} D_{s+}^{\alpha} Z[r] D_{t-}^{1-\alpha} \omega_{t-}[r] d r .
$$

We collect some properties of this integral; for the proof see [11] and [42].

Lemma 2.3. Let $Z, Z_{1}, Z_{2} \in C^{\beta}\left(\left[T_{1}, T_{2}\right] ; R\right), \omega, \omega_{1}, \omega_{2} \in C^{\beta^{\prime}}\left(\left[T_{1}, T_{2}\right] ; R\right)$ such that $\beta+\beta^{\prime}>1$. Then there exists a positive constant $C_{\beta, \beta^{\prime}}$ such that for $T_{1} \leq s<$ $t \leq T_{2}$

$$
\left|\int_{s}^{t} Z d \omega\right| \leq C_{\beta, \beta^{\prime}}\left(1+(t-s)^{\beta}\right)(t-s)^{\beta^{\prime}}\|Z\|_{\beta, T_{1}, T_{2}}\|\omega\|_{\beta^{\prime}, T_{1}, T_{2}} .
$$

In addition,

$$
\begin{aligned}
& \int_{s}^{t}\left(Z_{1}+Z_{2}\right) d \omega=\int_{s}^{t} Z_{1} d \omega+\int_{s}^{t} Z_{2} d \omega, \\
& \int_{s}^{t} Z d\left(\omega_{1}+\omega_{2}\right)=\int_{s}^{t} Z d \omega_{1}+\int_{s}^{t} Z d \omega_{2} .
\end{aligned}
$$

The integral is additive: for $\tau \in[s, t]$

$$
\int_{s}^{t} Z d \omega=\int_{s}^{\tau} Z d \omega+\int_{\tau}^{t} Z d \omega
$$

Copyright (c) by SIAM. Unauthorized reproduction of this article is prohibited. 
Moreover, for any $\tau \in R$

$$
\int_{s}^{t} Z(r) d \omega(r)=\int_{s-\tau}^{t-\tau} Z(r+\tau) d \theta_{\tau} \omega(r)
$$

where $\theta_{\tau} \omega(\cdot)=\omega(\cdot+\tau)-\omega(\tau)$. Finally, let $\left(\omega_{n}\right)_{n \in N}$ be a sequence converging in $C^{\beta^{\prime}}\left(\left[T_{1}, T_{2}\right] ; R\right)$ to $\omega$. Then we have

$$
\lim _{n \rightarrow \infty}\left\|\int_{T_{1}} Z d \omega_{n}-\int_{T_{1}}^{\cdot} Z d \omega\right\|_{\beta, T_{1}, T_{2}}=0
$$

Note that in the last expression, the integral with respect to $\omega_{n}$ can be interpreted in the Lebesgue sense.

We now extend the definition of a fractional integral in $R$ to a fractional integral in the separable Hilbert space $\ell^{2}$, following the construction carried out recently in [11] in a general separable Hilbert space. To do that, consider the separable Hilbert space $L_{2}\left(\ell^{2}\right)$ of Hilbert-Schmidt operators from $\ell^{2}$ into $\ell^{2}$, with the usual norm $\|\cdot\|_{L_{2}\left(\ell^{2}\right)}$ defined by

$$
\|z\|_{L_{2}\left(\ell^{2}\right)}^{2}=\sum_{i \in Z}\left\|z \mathbf{e}_{i}\right\|^{2}
$$

for $z \in L_{2}\left(\ell^{2}\right)$. Let $Z \in C^{\beta}\left(\left[T_{1}, T_{2}\right] ; L_{2}\left(\ell^{2}\right)\right)$ and $\omega \in C^{\beta^{\prime}}\left(\left[T_{1}, T_{2}\right] ; \ell^{2}\right)$ with $\beta+\beta^{\prime}>1$. We define the $\ell^{2}$-valued integral for $T_{1} \leq s<t \leq T_{2}$ as

$$
\int_{s}^{t} Z d \omega:=(-1)^{\alpha} \sum_{j \in Z}\left(\sum_{i \in Z} \int_{s}^{t} D_{s+}^{\alpha}\left\langle\mathbf{e}_{j}, Z(\cdot) \mathbf{e}_{i}\right\rangle[r] D_{t-}^{1-\alpha}\left\langle\mathbf{e}_{i}, \omega(\cdot)\right\rangle_{t-}[r] d r\right) \mathbf{e}_{j}
$$

for $1-\beta^{\prime}<\alpha<\beta$, whose norm fulfills

$$
\left\|\int_{s}^{t} Z d \omega\right\| \leq \int_{s}^{t}\left\|D_{s+}^{\alpha} Z[r]\right\|_{L_{2}\left(\ell^{2}\right)}\left\|D_{t-}^{1-\alpha} \omega_{t-}[r]\right\| d r .
$$

Note that in (2.2) the integrals under the sums are one-dimensional fractional integrals. In particular, in [11] the following result was proved.

Theorem 2.4. Suppose that $Z \in C^{\beta}\left(\left[T_{1}, T_{2}\right] ; L_{2}\left(\ell^{2}\right)\right)$ and $\omega \in C^{\beta^{\prime}}\left(\left[T_{1}, T_{2}\right] ; \ell^{2}\right)$, where $\beta+\beta^{\prime}>1$. Then there exists $\alpha \in(0,1)$ such that $1-\beta^{\prime}<\alpha<\beta$ and the integral (2.2) is well-defined. Moreover, all properties of Lemma 2.3 hold if we replace the $R$-norm by the $\ell^{2}$-norm.

We now consider estimates of the integral with respect to the Hölder norms depending on $\rho$.

Lemma 2.5. Under the assumptions of Theorem 2.4, for $\beta^{\prime}>\beta$ there exists a constant $c$ depending on $T_{1}, T_{2}, \beta, \beta^{\prime}$ such that for $T_{1} \leq s<t \leq T_{2}$

$$
e^{-\rho t}\left\|\int_{s}^{t} Z d \omega\right\| \leq c k(\rho)\|Z\|_{\beta, \rho, s, t}\|\omega\|_{\beta^{\prime}, s, t}(t-s)^{\beta},
$$

where $k(\rho)$ defined by (2.4) below fulfills $\lim _{\rho \rightarrow \infty} k(\rho)=0$.

Proof. We only sketch the proof; for more details see [11].

First of all, it is not difficult to see that

$$
\left\|D_{t-}^{1-\alpha} \omega_{t-}[r]\right\| \leq c\|\omega\|_{\beta^{\prime}, s, t}(t-r)^{\alpha+\beta^{\prime}-1}
$$

Copyright (c) by SIAM. Unauthorized reproduction of this article is prohibited. 
Furthermore, since $Z \in C^{\beta}\left(\left[T_{1}, T_{2}\right] ; L_{2}\left(\ell^{2}\right)\right)$,

$$
\begin{aligned}
e^{-\rho t}\left\|D_{s+}^{\alpha} Z[r]\right\|_{L_{2}\left(\ell^{2}\right)} & \leq c e^{-\rho(t-r)}\left(e^{-\rho r} \frac{\|Z(r)\|_{L_{2}\left(\ell^{2}\right)}}{(r-s)^{\alpha}}+\int_{s}^{r} e^{-\rho r} \frac{\|Z(r)-Z(q)\|_{L_{2}\left(\ell^{2}\right)}}{(r-q)^{1+\alpha}} d q\right) \\
& \leq c e^{-\rho(t-r)}\left(1+(r-s)^{\beta}\right)\|Z\|_{\beta, \rho, s, t}(r-s)^{-\alpha} \\
& \leq c e^{-\rho(t-r)}\|Z\|_{\beta, \rho, s, t}(r-s)^{-\alpha} .
\end{aligned}
$$

Therefore,

$$
\begin{aligned}
e^{-\rho t}\left\|\int_{s}^{t} Z d \omega\right\| & \leq c\|\omega\|_{\beta^{\prime}, s, t}\|Z\|_{\beta, \rho, s, t} \int_{s}^{t} e^{-\rho(t-r)}(t-r)^{\alpha+\beta^{\prime}-1}(r-s)^{-\alpha} d r \\
& \leq c\|\omega\|_{\beta^{\prime}, s, t}\|Z\|_{\beta, \rho, s, t}(t-s)^{\beta} \int_{s}^{t} e^{-\rho(t-r)}(t-r)^{\alpha+\beta^{\prime}-\beta-1}(r-s)^{-\alpha} d r \\
& \leq c k(\rho)\|\omega\|_{\beta^{\prime}, s, t}\|Z\|_{\beta, \rho, s, t}(t-s)^{\beta},
\end{aligned}
$$

where

$$
k(\rho)=\sup _{0 \leq s<t \leq T} \int_{s}^{t} e^{-\rho(t-r)}(t-r)^{\alpha+\beta^{\prime}-\beta-1}(r-s)^{-\alpha} d r
$$

is such that $\lim _{\rho \rightarrow \infty} k(\rho)=0$. The previous property can be stated in general as follows: given $T>0$, if $a, b>-1$ are such that $a+b+1>0$, then

$$
k(\rho):=\sup _{0 \leq s<t \leq T} \int_{s}^{t} e^{-\rho(t-r)}(r-s)^{a}(t-r)^{b} d r
$$

is such that $\lim _{\rho \rightarrow \infty} k(\rho)=0$; see [11].

From now on $k(\rho)$ will denote a function with the above behavior no matter the exact values of the corresponding parameters $a, b>-1$ provided that $a+b+1>0$. Moreover, note that the constraints in Lemma 2.5 imply that $\beta^{\prime}>1 / 2$.

As a particular case of Hölder-continuous integrator we are going to consider an $\mathrm{fBm}$ with values in $\ell^{2}$ and Hurst parameter $H>1 / 2$. Consider a probability space $(\Omega, \mathcal{F}, P)$. Let $\left(B_{i}^{H}\right)_{i \in Z}$ be an independent and identically distributed sequence of fBm with the same Hurst parameter $H>1 / 2$ over this probability space, that is, each $B_{i}^{H}$ is a centered Gauss process on $R$ with covariance

$$
\mathcal{R}(s, t)=\frac{1}{2}\left(|s|^{2 H}+|t|^{2 H}-|t-s|^{2 H}\right) \quad \text { for } s, t \in R .
$$

Let $Q$ be a linear operator on $\ell^{2}$ such that $Q \mathbf{e}_{i}=\sigma_{i}^{2} \mathbf{e}_{i}, \sigma=\left(\sigma_{i}\right)_{i \in Z}$. Hence $Q$ is a nonnegative and symmetric trace-class operator. A continuous $\ell^{2}$-valued $\mathrm{fBm} B^{H}$ with covariance operator $Q$ and Hurst parameter $H$ is defined by

$$
B^{H}(t)=\sum_{i \in Z}\left(\sigma_{i} B_{i}^{H}(t)\right) \mathbf{e}_{i}
$$

having covariance

$$
\mathcal{R}_{Q}(s, t)=\frac{1}{2} Q\left(|s|^{2 H}+|t|^{2 H}-|t-s|^{2 H}\right) \quad \text { for } s, t \in R .
$$

Copyright $@$ by SIAM. Unauthorized reproduction of this article is prohibited. 
In fact, since $B^{H}$ is a Gauss process,

$$
\begin{aligned}
& E\left\|B^{H}(t)-B^{H}(s)\right\|^{2}=\sum_{i \in Z} \sigma_{i}^{2} E\left(B_{i}^{H}(t)-B_{i}^{H}(s)\right)^{2}=\sum_{i \in Z} \sigma_{i}^{2}|t-s|^{2 H}=\|\sigma\|^{2}|t-s|^{2 H}, \\
& E\left\|B^{H}(t)-B^{H}(s)\right\|^{2 n} \leq c_{n}|t-s|^{2 H n} .
\end{aligned}
$$

Therefore, applying Kunita [32, Theorem 1.4.1], $B^{H}(t)$ has a continuous version and also a Hölder-continuous version with exponent $\beta^{\prime}<H$; see Bauer [4, Chapter 39]. Note that $B^{H}(0)=0$ almost surely.

Let $C_{0}\left(R ; \ell^{2}\right)$ be the space of continuous functions on $R$ with values in $\ell^{2}$ which are zero at zero, equipped with the compact open topology. Consider the canonical space for the $\mathrm{fBm}\left(C_{0}\left(R ; \ell^{2}\right), \mathcal{B}\left(C_{0}\left(R ; \ell^{2}\right)\right), P_{H}\right)$, where $B^{H}(\omega)=\omega$ and $P_{H}$ denotes the measure of the fBm with Hurst parameter $H$. On $C_{0}\left(R ; \ell^{2}\right)$ we can introduce the Wiener shift $\theta$ given by the measurable flow

$$
\theta:\left(R \times C_{0}\left(R, \ell^{2}\right), \mathcal{B}(R) \otimes \mathcal{B}\left(C_{0}\left(R, \ell^{2}\right)\right)\right) \rightarrow\left(C_{0}\left(R, \ell^{2}\right), \mathcal{B}\left(C_{0}\left(R, \ell^{2}\right)\right)\right)
$$

such that

$$
\theta(t, \omega)(\cdot)=\theta_{t} \omega(\cdot)=\omega(\cdot+t)-\omega(t)
$$

By Mishura [35, p. 8], we have that $\theta_{t}$ leaves $P_{H}$ invariant. In addition $t \rightarrow \theta_{t} \omega$ is continuous. Furthermore, thanks to Bauer [4, Chapter 39], we can also conclude that the set $C_{0}^{\beta^{\prime}}\left(R ; \ell^{2}\right)$ of continuous functions which have a finite $\beta^{\prime}$-Hölder-seminorm on any compact interval and which are zero at zero has $P_{H}$-measure one for $\beta^{\prime}<H$. This set is $\theta$-invariant.

3. Lattice equations driven by fractional Brownian motions. Given strictly positive constants $\nu$ and $\lambda$, we consider the following SLDS with a diffusive adjacent neighborhood interaction, a dissipative nonlinear reaction term, and an $\mathrm{fBm} B_{i}^{H}$ at each node:

$$
d u_{i}(t)=\left(\nu\left(u_{i-1}-2 u_{i}+u_{i+1}\right)-\lambda u_{i}+f_{i}\left(u_{i}\right)\right) d t+\sigma_{i} h_{i}\left(u_{i}\right) d B_{i}^{H}(t), i \in Z .
$$

Here $f_{i}$ and $h_{i}$ are suitable regular functions; see below. We want to rewrite this system giving it the interpretation of a stochastic evolution equation in $\ell^{2}$. To this end, let $A$ be the linear bounded operator from $\ell^{2}$ to $\ell^{2}$ defined by $A u=\left((A u)_{i}\right)_{i \in Z}$, where

$$
(A u)_{i}=-\nu\left(u_{i-1}-2 u_{i}+u_{i+1}\right), \quad i \in Z .
$$

Notice that $A=B B^{*}=B^{*} B$, where

$$
(B u)_{i}=\sqrt{\nu}\left(u_{i+1}-u_{i}\right), \quad\left(B^{*} u\right)_{i}=\sqrt{\nu}\left(u_{i-1}-u_{i}\right)
$$

and hence

$$
\langle A u, u\rangle \geq 0 \quad \forall u \in \ell^{2} .
$$

Let us consider the linear bounded operator $A_{\lambda}: \ell^{2} \rightarrow \ell^{2}$ given by

$$
A_{\lambda} u=A u+\lambda u .
$$

Copyright (C) by SIAM. Unauthorized reproduction of this article is prohibited. 
Then

$$
\left\langle A_{\lambda} u, u\right\rangle \geq \lambda\|u\|^{2} \quad \forall u \in \ell^{2}
$$

hence $-A_{\lambda}$ is a negative defined and bounded operator, and thus it generates a uniformly continuous (semi)group $S_{\lambda}:=e^{-A_{\lambda} t}$ on $\ell^{2}$, for which the following estimates hold true.

Lemma 3.1. The uniformly continuous semigroup $S_{\lambda}$ is also exponentially stable, that is, for $t \geq 0$ we have

$$
\left\|S_{\lambda}(t)\right\|_{L\left(\ell^{2}\right)} \leq e^{-\lambda t}
$$

In addition, for $0 \leq s \leq t$

$$
\begin{aligned}
\left\|S_{\lambda}(t-s)-\mathrm{id}\right\|_{L\left(\ell^{2}\right)} & \leq\left\|A_{\lambda}\right\|(t-s), \\
\left\|S_{\lambda}(t)-S_{\lambda}(s)\right\|_{L\left(\ell^{2}\right)} & \leq\left\|A_{\lambda}\right\|(t-s) e^{-\lambda s},
\end{aligned}
$$

where, for the sake of presentation, $\left\|A_{\lambda}\right\|$ represents $\left\|A_{\lambda}\right\|_{L\left(\ell^{2}\right)}\left(L\left(\ell^{2}\right)\right.$ denotes the space of linear continuous operators from $\ell^{2}$ into itself).

The proof of the first property is a direct consequence of the energy inequality, while the two last estimates follow easily by the mean value theorem. As straightforward results, we also obtain that for $0<s<t$,

$$
\left\|S_{\lambda}(t-\cdot)\right\|_{\beta, 0, t}=\sup _{0 \leq r_{1}<r_{2} \leq t} \frac{\left\|S_{\lambda}\left(t-r_{2}\right)-S_{\lambda}\left(t-r_{1}\right)\right\|_{L\left(\ell^{2}\right)}}{\left(r_{2}-r_{1}\right)^{\beta}} \leq\left\|A_{\lambda}\right\| t^{1-\beta},
$$

and

$$
\begin{aligned}
& \left\|S_{\lambda}(t-\cdot)-S_{\lambda}(s-\cdot)\right\|_{\beta, 0, s} \\
= & \sup _{0 \leq r_{1}<r_{2} \leq s} \frac{\left\|\left(S_{\lambda}(t-s)-\mathrm{id}\right)\left(S_{\lambda}\left(s-r_{2}\right)-S_{\lambda}\left(s-r_{1}\right)\right)\right\|_{L\left(\ell^{2}\right)}}{\left(r_{2}-r_{1}\right)^{\beta}} \\
\leq & \left\|A_{\lambda}\right\|^{2}(t-s) s^{1-\beta} .
\end{aligned}
$$

Now we formulate the assumptions for the functions $f_{i}$ and $g_{i}$. Indeed, for the sake of completeness, we present now all the standing assumptions needed in this section:

(A1) The process $\omega$ is a (canonical) continuous fBm with values in $\ell^{2}$, with covariance $Q$, and with Hurst parameter $H$ given by (2.6). In particular, we have parameters $\frac{1}{2}<\beta<\beta^{\prime}<H$ and $1-\beta^{\prime}<\alpha<\beta$.

(A2) Let $A_{\lambda}$ be the operator defined by (3.2) and $S_{\lambda}$ be the exponentially stable and uniformly continuous semigroup generated by $-A_{\lambda}$.

(A3) $f_{i} \in C^{1}(R, R), \sum_{i \in Z} f_{i}(0)^{2}<\infty$, and there exists a constant $D_{f} \geq 0$ such that

$$
\left|f_{i}^{\prime}(\zeta)\right| \leq D_{f}, \quad \zeta \in R, i \in Z
$$

(A4) $h_{i} \in C^{2}(R, R), \sum_{i \in Z} h_{i}(0)^{2}<\infty$, and there exist constants $D_{h}, M_{h} \geq 0$ such that

$$
\left|h_{i}^{\prime}(\zeta)\right| \leq D_{h}, \quad\left|h_{i}^{\prime \prime}(\zeta)\right| \leq M_{h}, \quad \zeta \in R, i \in Z
$$

Copyright (c) by SIAM. Unauthorized reproduction of this article is prohibited. 
Let $u=\left(u_{i}\right)_{i \in Z}$ be an element of $\ell^{2}$. Then (A3) allows us to define the operator

$$
f: \ell^{2} \rightarrow \ell^{2}, \quad f(u):=\left(f_{i}\left(u_{i}\right)\right)_{i \in Z} .
$$

Thanks to (A4) we can also define the operator $h(u) \in L\left(\ell^{2}\right)$ by

$$
h(u) v=\left(h_{i}\left(u_{i}\right) v_{i}\right)_{i \in Z} \in \ell^{2} .
$$

That $f$ and $h$ are well-posed is proved in the next result, as well as their main regularity properties.

Lemma 3.2. (a) The operator $f: \ell^{2} \rightarrow \ell^{2}$ given by (3.7) is well-defined and is Lipschitz continuous with Lipschitz constant $D_{f}$.

(b) The operator $\ell^{2} \ni u \mapsto h(u) \in L_{2}\left(\ell^{2}\right)$ given by (3.8) is well-defined and continuously differentiable. Moreover, both $h$ and its first derivative Dh are Lipschitzcontinuous with Lipschitz constants $D_{h}$ and $M_{h}$, respectively. Furthermore, for $u, v$, $w, z \in \ell^{2}$ the following property holds true:

$$
\begin{aligned}
\| h(u)-h(v) & -(h(w)-h(z))\left\|_{L_{2}\left(\ell^{2}\right)} \leq \sqrt{2} D_{h}\right\| u-v-(w-z) \| \\
& +2 M_{h}\|u-w\|(\|u-v\|+\|w-z\|) .
\end{aligned}
$$

Proof. (a) Thanks to the definition of $f$, for $u=\left(u_{i}\right)_{i \in Z} \in \ell^{2}$ we have

$$
\|f(u)\|^{2} \leq 2 \sum_{i \in Z} f_{i}(0)^{2}+2 D_{f}^{2}\|u\|^{2}<\infty,
$$

hence it is well-posed. Furthermore, $f$ is Lipschitz-continuous: for $v=\left(v_{i}\right)_{i \in Z} \in \ell^{2}$ we obtain

$$
\|f(u)-f(v)\|^{2}=\sum_{i \in Z}\left|f_{i}\left(u_{i}\right)-f_{i}\left(v_{i}\right)\right|^{2} \leq D_{f}^{2} \sum_{i \in Z}\left|u_{i}-v_{i}\right|^{2}=D_{f}^{2}\|u-v\|^{2} .
$$

(b) The operator $h$ is well-defined as a Hilbert-Schmidt operator, since

$$
\begin{gathered}
\|h(u)\|_{L_{2}\left(\ell^{2}\right)}^{2}=\sum_{i \in Z}\left\|h(u) \mathbf{e}_{i}\right\|^{2}=\sum_{i, j \in Z}\left|\left(h(u) \mathbf{e}_{i}\right)_{j}\right|^{2}=\sum_{i \in Z}\left|h_{i}\left(u_{i}\right)\right|^{2} \\
\leq 2 \sum_{i \in Z} h_{i}(0)^{2}+2 D_{h}^{2}\|u\|^{2}<\infty .
\end{gathered}
$$

Moreover, in a similar way to how we have proceeded for the operator $f, h$ is Lipschitzcontinuous:

$$
\|h(u)-h(v)\|_{L_{2}\left(\ell^{2}\right)}^{2} \leq D_{h}^{2}\|u-v\|^{2} \quad \text { for } u, v \in \ell^{2} .
$$

Regarding the derivative, we have that $D h: \ell^{2} \mapsto L\left(\ell^{2}, L_{2}\left(\ell^{2}\right)\right)$ is defined for $u, v, w \in$ $\ell^{2}$ by

$$
(D h(u) v) w=\left(h_{i}^{\prime}\left(u_{i}\right) v_{i} w_{i}\right)_{i \in Z}
$$

In fact,

$$
\begin{array}{r}
\|h(u+v)-h(u)-D h(u) v\|_{L_{2}\left(\ell^{2}\right)}^{2}=\sum_{i \in Z}\left|h_{i}\left(u_{i}+v_{i}\right)-h_{i}\left(u_{i}\right)-h_{i}^{\prime}\left(u_{i}\right) v_{i}\right|^{2} \\
\quad \leq \frac{1}{4} \sum_{i \in Z}\left|h_{i}^{\prime \prime}\left(\tilde{u}_{i}\right)\right|^{2} v_{i}^{4} \leq \frac{1}{4} M_{h}^{2} \sum_{i \in Z} v_{i}^{4} \leq \frac{1}{4} M_{h}^{2}\left(\sum_{i \in Z} v_{i}^{2}\right)^{2} \leq \frac{1}{4} M_{h}^{2}\|v\|^{4},
\end{array}
$$

Copyright (c) by SIAM. Unauthorized reproduction of this article is prohibited. 
where $\tilde{u}_{i}$ is an intermediate element between $u_{i}$ and $u_{i}+v_{i}$. This derivative is bounded in the space $L\left(\ell^{2}, L_{2}\left(\ell^{2}\right)\right)$ since

$$
\|D h(u)\|_{L\left(\ell^{2}, L_{2}\left(\ell^{2}\right)\right)}^{2}=\sup _{\|z\|=1} \sum_{i \in Z}\left|h_{i}^{\prime}\left(u_{i}\right) z_{i}\right|^{2} \leq D_{h}^{2},
$$

and furthermore $D h$ is Lipschitz-continuous:

$$
\begin{gathered}
\|D h(u)-D h(v)\|_{L\left(\ell^{2}, L_{2}\left(\ell^{2}\right)\right)}^{2}=\sup _{\|z\|=1} \sum_{i \in Z}\left|h_{i}^{\prime}\left(u_{i}\right) z_{i}-h_{i}^{\prime}\left(v_{i}\right) z_{i}\right|^{2} \\
\leq \sup _{\|z\|=1} \sum_{i \in Z}\left|h_{i}^{\prime \prime}\left(\tilde{u}_{i}\right)\left(u_{i}-v_{i}\right) z_{i}\right|^{2} \leq M_{h}^{2}\|u-v\|^{2} .
\end{gathered}
$$

Finally, property (3.9) follows by Nualart and Răşcanu [36, Lemma 7.1]. Indeed, by virtue of the Lispchitz continuity of any $h_{i}$ and $h_{i}^{\prime}$ we obtain

$$
\begin{aligned}
& \|h(u)-h(v)-(h(w)-h(z))\|_{L_{2}\left(\ell^{2}\right)}^{2}=\sum_{i \in Z}\left|h_{i}\left(u_{i}\right)-h_{i}\left(v_{i}\right)-\left(h_{i}\left(w_{i}\right)-h_{i}\left(z_{i}\right)\right)\right|^{2} \\
& \quad \leq \sum_{i \in Z}\left(2 D_{h}^{2}\left|u_{i}-v_{i}-\left(w_{i}-z_{i}\right)\right|^{2}+4 M_{h}^{2}\left|u_{i}-w_{i}\right|^{2}\left(\left|u_{i}-v_{i}\right|^{2}+\left|w_{i}-z_{i}\right|^{2}\right)\right) \\
& \quad \leq 2 D_{h}^{2}\|u-v-(w-z)\|^{2}+4 M_{h}^{2}\|u-w\|^{2}\left(\|u-v\|^{2}+\|w-z\|^{2}\right) .
\end{aligned}
$$

Hence, we can reformulate the system of equations given by (3.1) as the following evolution equation with values in $\ell^{2}$ :

$$
d u(t)=\left(-A_{\lambda} u(t)+f(u(t))\right) d t+h(u(t)) d \omega(t),
$$

where $A_{\lambda}$ has been defined by (3.2), and $f$ and $h$ by (3.7) and (3.8), respectively. The sequence $u(t)=\left(u_{i}(t)\right)_{i \in Z}$ is such that $u_{i}$ fulfills (3.1) for each $i \in Z$. Since our stability considerations will be based on the exponential stability of $S_{\lambda}$, we look for a mild solution of the above equation, namely, we look for an $u(t)=\left(u_{i}(t)\right)_{i \in Z} \in \ell^{2}$ solution of the operator equation

$$
u(t)=S_{\lambda}(t) x+\int_{0}^{t} S_{\lambda}(t-r) f(u(r)) d r+\int_{0}^{t} S_{\lambda}(t-r) h(u(r)) d \omega(r),
$$

where the initial condition $x \in \ell^{2}$. The last integral has to be interpreted as we have explained in section 2 .

Next we would like to apply a fixed point argument to ensure the existence and uniqueness of a solution to (3.10). We first present estimates of the stochastic integral appearing on the right-hand side of (3.10).

Lemma 3.3. Under assumptions (A1), (A2), and (A4), the stochastic integral satisfies

$$
\left\|\int_{0}^{\cdot} S_{\lambda}(\cdot-r) h(u(r)) d \omega(r)\right\|_{\beta, \rho, 0, T} \leq c k(\rho)\|\omega\|_{\beta^{\prime}, 0, T}\|h(u(\cdot))\|_{\beta, \rho, 0, T},
$$

where $c$ may depend on $\beta, \beta^{\prime}, T,\left\|A_{\lambda}\right\|$, and $k(\rho)$ is given by (2.5). Furthermore,

$$
\begin{aligned}
\left\|\int_{0} S_{\lambda}(\cdot-r) h(u(r)) d \omega(r)\right\|_{\infty, 0, T} & \leq c\left(1+\left\|A_{\lambda}\right\|\right)\|\omega\|_{\beta^{\prime}, 0, T}\|h(u(\cdot))\|_{\beta, 0, T}, \\
\left\|\int_{0} S_{\lambda}(\cdot-r) h(u(r)) d \omega(r)\right\|_{\beta, 0, T} & \leq c\left(1+\left\|A_{\lambda}\right\|\right)^{2}\|\omega\|_{\beta^{\prime}, 0, T}\|h(u(\cdot))\|_{\beta, 0, T},
\end{aligned}
$$

where in the last two inequalities $c$ may depend on $\beta, \beta^{\prime}$, and $T$.

Copyright $@$ by SIAM. Unauthorized reproduction of this article is prohibited. 
Proof. Thanks to the additivity of the stochastic integral we can consider the following splitting:

$$
\begin{aligned}
\int_{0}^{t} & S_{\lambda}(t-r) h(u(r)) d \omega(r)-\int_{0}^{s} S_{\lambda}(s-r) h(u(r)) d \omega(r) \\
& =\int_{s}^{t} S_{\lambda}(t-r) h(u(r)) d \omega(r)+\int_{0}^{s}\left(S_{\lambda}(t-r)-S_{\lambda}(s-r)\right) h(u(r)) d \omega(r) .
\end{aligned}
$$

From (2.3), for $0 \leq s<t \leq T$ we obtain

$$
\begin{array}{r}
e^{-\rho t} \frac{\left\|\int_{s}^{t} S_{\lambda}(t-r) h(u(r)) d \omega(r)\right\|}{(t-s)^{\beta}} \leq c k(\rho)\|\omega\|_{\beta^{\prime}, 0, T}\left\|S_{\lambda}(t-\cdot) h(u(\cdot))\right\|_{\beta, \rho, 0, t}, \\
e^{-\rho t} \frac{\left\|\int_{0}^{s}\left(S_{\lambda}(t-r)-S_{\lambda}(s-r)\right) h(u(r)) d \omega(r)\right\|}{(t-s)^{\beta}} \leq c k(\rho)\|\omega\|_{\beta^{\prime}, 0, T} \frac{s^{\beta}}{(t-s)^{\beta}} \\
\times\left\|\left(S_{\lambda}(t-\cdot)-S_{\lambda}(s-\cdot)\right) h(u(\cdot))\right\|_{\beta, \rho, 0, s} .
\end{array}
$$

Furthermore, since for any two $\beta$-Hölder-continuous functions $l, g$ we easily obtain

$$
\|l g\|_{\beta, \rho, 0, t} \leq\|l\|_{\infty, 0, t}\|g\|_{\beta, \rho, 0, t}+\|g\|_{\infty, \rho, 0, t}\|l\|_{\beta, 0, t},
$$

by (3.3) and (3.5) we derive

$$
\begin{aligned}
& \left\|S_{\lambda}(t-\cdot) h(u(\cdot))\right\|_{\beta, \rho, 0, t} \\
& \quad \leq\left\|S_{\lambda}(t-\cdot)\right\|_{\infty, 0, t}\|h(u(\cdot))\|_{\beta, \rho, 0, t}+\left\|S_{\lambda}(t-\cdot)\right\|_{\beta, 0, t}\|h(u(\cdot))\|_{\infty, \rho, 0, t} \\
& \quad \leq\|h(u(\cdot))\|_{\beta, \rho, 0, t}+\left\|A_{\lambda}\right\| t^{1-\beta}\|h(u(\cdot))\|_{\infty, \rho, 0, t},
\end{aligned}
$$

and by (3.4) and (3.6)

$$
\begin{aligned}
& \left\|\left(S_{\lambda}(t-\cdot)-S_{\lambda}(s-\cdot)\right) h(u(\cdot))\right\|_{\beta, \rho, 0, s} \\
& \quad \leq(t-s)\left\|A_{\lambda}\right\|\|h(u(\cdot))\|_{\beta, \rho, 0, s}+\left\|A_{\lambda}\right\|^{2}(t-s) s^{1-\beta}\|h(u(\cdot))\|_{\infty, \rho, 0, s} .
\end{aligned}
$$

Hence

$$
\left\|\int_{0} S_{\lambda}(\cdot-r) h(u(r)) d \omega(r)\right\|_{\beta, \rho, 0, T} \leq c k(\rho)\|\omega\|_{\beta^{\prime}, 0, T}\|h(u(\cdot))\|_{\beta, \rho, 0, T} .
$$

Taking into account the way in which we have estimated the first term on the righthand side of (3.14), we immediately obtain

$$
\left\|\int_{0}^{\cdot} S_{\lambda}(\cdot-r) h(u(r)) d \omega(r)\right\|_{\infty, \rho, 0, T} \leq c k(\rho)\|\omega\|_{\beta^{\prime}, 0, T}\|h(u(\cdot))\|_{\beta, \rho, 0, T},
$$

so the proof of (3.11) is complete.

Notice that using the $\beta$-norm, (3.15) reads as follows

$$
\|l g\|_{\beta, 0, t} \leq\|l\|_{\infty, 0, t}\|g\|_{\beta, 0, t}+\|g\|_{\infty, 0, t}\|l\|_{\beta, 0, t},
$$

hence (3.12) is an immediate consequence of (3.3) and (3.5). In order to prove (3.13) we can follow the same steps as at the beginning of this proof.

Copyright $@$ by SIAM. Unauthorized reproduction of this article is prohibited. 
Now we can establish the existence of a unique mild solution to our SLDS.

TheOREM 3.4. Under assumptions (A1)-(A4), for every $T>0$ and $x \in \ell^{2}$ the problem (3.10) has a unique solution $u(\cdot)=u(\cdot, \omega, x) \in C^{\beta}\left([0, T] ; \ell^{2}\right)$.

Proof. We will show that the operator

$$
\mathcal{T}_{x, \omega}(u)[t]=S_{\lambda}(t) x+\int_{0}^{t} S_{\lambda}(t-r) f(u(r)) d r+\int_{0}^{t} S_{\lambda}(t-r) h(u(r)) d \omega(r),
$$

where $t \in[0, T]$, has a unique fixed point in $C^{\beta}\left([0, T] ; \ell^{2}\right)$ by applying the Banach fixed point theorem. To this end, first of all we show that there exists a closed centered ball with respect to the norm $\|\cdot\|_{\beta, \rho, 0, T}$ which is mapped by $\mathcal{T}_{x, \omega}$ into itself. For the first term, in virtue of (3.4),

$$
\left\|S_{\lambda}(\cdot) x\right\|_{\beta, \rho, 0, T} \leq\left(1+\left\|A_{\lambda}\right\| T^{1-\beta}\right)\|x\| .
$$

For the Lebesgue integral of $\mathcal{T}_{x, \omega}$ we obtain

$$
\begin{aligned}
\left\|\int_{0} S_{\lambda}(\cdot-r) f(u(r)) d r\right\|_{\beta, \rho, 0, T} & \leq \sup _{t \in[0, T]} e^{-\rho t}\left\|\int_{0}^{t} S_{\lambda}(t-r) f(u(r)) d r\right\| \\
& +\sup _{0 \leq s<t \leq T} e^{-\rho t} \frac{\left\|\int_{s}^{t} S_{\lambda}(t-r) f(u(r)) d r\right\|}{(t-s)^{\beta}} \\
& +\sup _{0 \leq s<t \leq T} e^{-\rho t} \frac{\left\|\int_{0}^{s}\left(S_{\lambda}(t-r)-S_{\lambda}(s-r)\right) f(u(r)) d r\right\|}{(t-s)^{\beta}} \\
& \leq \tilde{k}(\rho)\|f(u(\cdot))\|_{\infty, \rho, 0, T},
\end{aligned}
$$

where $\lim _{\rho \rightarrow \infty} \tilde{k}(\rho)=0$. In fact, we are going to show that

$$
\tilde{k}(\rho)=\left(\frac{1}{\rho}+c_{\beta} \frac{1}{\rho^{1-\beta}}+\frac{1}{\rho} T^{1-\beta}\left\|A_{\lambda}\right\|\right),
$$

where $c_{\beta}$ is a positive constant depending on $\beta$. Note that the first term on the right-hand side of (3.16) is estimated by

$$
\sup _{t \in[0, T]} \int_{0}^{t} e^{-\rho(t-r)} d r\|f(u(\cdot))\|_{\infty, \rho, 0, T} \leq \frac{1}{\rho}\|f(u(\cdot))\|_{\infty, \rho, 0, T} .
$$

For the second expression,

$$
\frac{\int_{s}^{t} e^{-\rho(t-r)} d r}{(t-s)^{\beta}} \leq \frac{1}{\rho^{1-\beta}} \frac{1-e^{-\rho(t-s)}}{\rho^{\beta}(t-s)^{\beta}} \leq \frac{1}{\rho^{1-\beta}} \sup _{x>0} \frac{1-e^{-x}}{x^{\beta}}=: \frac{1}{\rho^{1-\beta}} c_{\beta} .
$$

The estimate of the last term on the right-hand side of (3.16) follows by (3.4), since $S_{\lambda}(t-r)-S_{\lambda}(s-r)=\left(S_{\lambda}(t-s)-\mathrm{Id}\right) S_{\lambda}(s-r)$. On the other hand,

$$
\begin{aligned}
\|f(u(\cdot))\|_{\infty, \rho, 0, T} & \leq \sup _{0 \leq t \leq T} e^{-\rho t}\|f(x)\|+\sup _{0 \leq t \leq T} e^{-\rho t}\|f(u(t))-f(x)\| \\
& \leq\|f(x)\|+D_{f} T^{\beta}\|u\|_{\beta, \rho, 0, T},
\end{aligned}
$$

Copyright $@$ by SIAM. Unauthorized reproduction of this article is prohibited. 
hence

$$
\left\|\int_{0}^{\cdot} S_{\lambda}(\cdot-r) f(u(r)) d r\right\|_{\beta, \rho, 0, T} \leq \hat{k}(\rho)\left(1+\|u\|_{\beta, \rho, 0, T}\right)
$$

where now $\hat{k}(\rho)=\max \left\{\|f(x)\|, D_{f} T^{\beta}\right\} \tilde{k}(\rho)$, with $\tilde{k}(\rho)$ defined by (3.17).

On the other hand,

$$
\begin{aligned}
\|h(u(\cdot))\|_{\beta, \rho, 0, t} & =\sup _{r \in[0, t]} e^{-\rho r}\|h(u(r))\|_{L_{2}\left(\ell^{2}\right)} \\
& +\sup _{0 \leq q<r \leq t} \frac{e^{-\rho r}\|h(u(r))-h(u(q))\|_{L_{2}\left(\ell^{2}\right)}}{(r-q)^{\beta}} \\
& \leq\|h(x)\|+D_{h}\left(1+T^{\beta}\right)\|u\|_{\beta, \rho, 0, T}
\end{aligned}
$$

hence, on account of (3.11) we obtain

$$
\left\|\int_{0} S_{\lambda}(\cdot-r) h(u(r)) d \omega(r)\right\|_{\beta, \rho, 0, T} \leq c k(\rho)\|\omega\|_{\beta^{\prime}, 0, T}\left(1+\|u\|_{\beta, \rho, 0, T}\right),
$$

where $c$ may depend on $\beta, \beta^{\prime}, T,\left\|A_{\lambda}\right\|,\|h(x)\|$, and $D_{h}$. In conclusion, we have obtained

$$
\left\|\mathcal{T}_{x, \omega}(u)\right\|_{\beta, \rho, 0, T} \leq\left(1+\left\|A_{\lambda}\right\| T^{1-\beta}\right)\|x\|+K(\rho)\left(1+\|\omega\|_{\beta^{\prime}, 0, T}\right)\left(1+\|u\|_{\beta, \rho, 0, T}\right),
$$

where $\lim _{\rho \rightarrow \infty} K(\rho)=0$. Note that $K(\rho)$ may also depend on the parameters related to $f$ and $h$, the initial condition $x,\left\|A_{\lambda}\right\|$, and $T$. Taking a sufficiently large $\rho$ such that $K(\rho)\left(1+\|\omega\|_{\beta^{\prime}, 0, T}\right) \leq 1 / 2$, the ball

$$
B=B(0, R(x, \rho))=\left\{u \in C^{\beta}\left([0, T] ; \ell^{2}\right):\|u\|_{\beta, \rho, 0, T} \leq R\right\}
$$

with

$$
R=R(x, \rho)=2\left(1+\left\|A_{\lambda}\right\| T^{1-\beta}\right)\|x\|+1
$$

is mapped into itself since

$$
\left\|\mathcal{T}_{x, \omega}(u)\right\|_{\beta, \rho, 0, T} \leq\left(1+\left\|A_{\lambda}\right\| T^{1-\beta}\right)\|x\|+\frac{1}{2}(1+R)=R .
$$

We now derive the contraction condition for the operator $\mathcal{T}_{x, \omega}$ with respect to the norm $\|\cdot\|_{\beta, \bar{\rho}, 0, T}$, where the $\bar{\rho}$ may differ from the $\rho$ considered above. However, since all these norms are equivalent for different $\rho \geq 0$, the ball $B$ remains a complete space with respect to any $\|\cdot\|_{\beta, \bar{\rho}, 0, T}$.

Similar to above, for the Lebesgue integral we obtain the estimate

$$
\left\|f\left(u_{1}(\cdot)\right)-f\left(u_{2}(\cdot)\right)\right\|_{\beta, \bar{\rho}, 0, T} \leq \tilde{k}(\bar{\rho}) D_{f}\left\|u_{1}-u_{2}\right\|_{\beta, \bar{\rho}, 0, T},
$$

where $\tilde{k}(\rho)$ is defined by (3.17) replacing $\rho$ by $\bar{\rho}$.

Regarding the stochastic integral, the difference with respect to the previous computations is that now in (3.18) the operator $h(u(\cdot))$ has to be replaced by $h\left(u_{1}(\cdot)\right)$ $h\left(u_{2}(\cdot)\right)$. In particular, from $(3.9)$ we easily derive

$$
\begin{aligned}
\left\|h\left(u_{1}(\cdot)\right)-h\left(u_{2}(\cdot)\right)\right\|_{\beta, \bar{\rho}, 0, T} & \leq D_{h}\left(\left\|u_{1}-u_{2}\right\|_{\infty, \bar{\rho}, 0, T}+\sqrt{2}\left\|u_{1}-u_{2}\right\|_{\beta, \bar{\rho}, 0, T}\right) \\
& +2 M_{h}\left(\left\|u_{1}\right\|_{\beta, 0, T}+\left\|u_{2}\right\|_{\beta, 0, T}\right)\left\|u_{1}-u_{2}\right\|_{\infty, \bar{\rho}, 0, T} .
\end{aligned}
$$

Copyright $@$ by SIAM. Unauthorized reproduction of this article is prohibited. 
Since $\left\|u_{1}\right\|_{\beta, 0, T} \leq e^{\rho T} R(x, \rho)$ (and the same inequality holds for $u_{2}$ ), then

$$
\begin{array}{r}
\left\|\mathcal{T}_{x, \omega}\left(u_{1}\right)-\mathcal{T}_{x, \omega}\left(u_{2}\right)\right\|_{\beta, \bar{\rho}, 0, T} \leq K(\bar{\rho})\left(1+2 e^{\rho T} R(x, \rho)\right) \\
\times\left\|u_{1}-u_{2}\right\|_{\beta, \bar{\rho}, 0, T},
\end{array}
$$

where again $\lim _{\bar{\rho} \rightarrow \infty} K(\bar{\rho})=0$.. It suffices then to choose $\bar{\rho}$ sufficiently large so that

$$
\left\|\mathcal{T}_{x, \omega}\left(u_{1}\right)-\mathcal{T}_{x, \omega}\left(u_{2}\right)\right\|_{\beta, \bar{\rho}, 0, T} \leq \frac{1}{2}\left\|u_{1}-u_{2}\right\|_{\beta, \bar{\rho}, 0, T},
$$

which implies the contraction property of the map $T_{x, \omega}$. Hence, (3.10) has a unique solution $u \in B$. And by similar arguments there cannot be another solution outside the ball $\mathrm{B}$.

We finish this section by proving that the solution of (3.10) generates an RDS.

Definition 3.5. Consider a probability space $(\Omega, \mathcal{F}, P)$. The quadruple $(\Omega, \mathcal{F}, P, \theta)$ is called a metric dynamical system if the measurable mapping

$$
\theta:(R \times \Omega, \mathcal{B}(R) \otimes \mathcal{F}) \rightarrow(\Omega, \mathcal{F})
$$

is a flow, that is,

$$
\theta_{t_{1}} \circ \theta_{t_{2}}=\theta_{t_{1}} \theta_{t_{2}}=\theta_{t_{1}+t_{2}}, t_{1}, t_{2} \in R ; \quad \theta_{0}=\mathrm{id}_{\Omega},
$$

and the measure $P$ is invariant and ergodic with respect to $\theta$.

DEFINITION 3.6. An RDS $\varphi$ over the metric dynamical system $(\Omega, \mathcal{F}, P, \theta)$ is a $\left(\mathcal{B}\left(R^{+}\right) \otimes \mathcal{F} \otimes \mathcal{B}\left(\ell^{2}\right), \mathcal{B}\left(\ell^{2}\right)\right)$-measurable mapping such that the cocycle property holds

$$
\varphi(t+\tau, \omega, x)=\varphi\left(t, \theta_{\tau} \omega, \varphi(\tau, \omega, x)\right), \quad \varphi(0, \omega, x)=x,
$$

for all $t \geq \tau \in R^{+}, x \in \ell^{2}$, and $\omega \in \Omega$.

The metric dynamical system is the model for the noise, in our case the $\mathrm{fBm}$. More precisely, we take the quadruple $(\Omega, \mathcal{F}, P, \theta)=\left(C_{0}\left(R ; \ell^{2}\right), \mathcal{B}\left(C_{0}\left(R ; \ell^{2}\right)\right), P_{H}, \theta\right)$, where $\theta$ is given by the Wiener flow introduced in (2.7).

TheOREM 3.7. The solution of (3.10) generates an $R D S$

$$
\varphi: R^{+} \times \Omega \times \ell^{2} \mapsto \ell^{2}
$$

given by $\varphi(t, \omega, x)=u(t, \omega, x)=u(t)$, where $u$ the unique solution to (3.10) corresponding to $\omega$ and initial condition $x$.

Proof. We only sketch the main ideas of the proof.

The cocycle property is a consequence in particular of the additivity of the stochastic integral as well as the behavior of the stochastic integral when performing a change of variable given by (2.1). More specifically,

$$
\begin{aligned}
\varphi(t+\tau, \omega, x)= & S_{\lambda}(t+\tau) x+\int_{0}^{t+\tau} S_{\lambda}(t+\tau-r) f(u(r)) d r \\
& +\int_{0}^{t+\tau} S_{\lambda}(t+\tau-r) h(u(r)) d \omega(r) \\
= & S_{\lambda}(t)\left(S_{\lambda}(\tau) x+\int_{0}^{\tau} S_{\lambda}(\tau-r) f(u(r)) d r+\int_{0}^{\tau} S_{\lambda}(\tau-r) h(u(r)) d \omega(r)\right) \\
& +\int_{\tau}^{t+\tau} S_{\lambda}(t+\tau-r) f(u(r)) d r+\int_{\tau}^{t+\tau} S_{\lambda}(t+\tau-r) h(u(r)) d \omega(r) \\
= & S(t) u(\tau)+\int_{0}^{t} S_{\lambda}(t-r) f(u(r+\tau)) d r+\int_{0}^{t} S_{\lambda}(t-r) h(u(r+\tau)) d \theta_{\tau} \omega(r) .
\end{aligned}
$$

Copyright $\odot$ by SIAM. Unauthorized reproduction of this article is prohibited. 
Denoting $y(\cdot)=u(\cdot+\tau)$ the previous inequality reads

$$
\varphi(t+\tau, \omega, x)=S_{\lambda}(t) y(0)+\int_{0}^{t} S_{\lambda}(t-r) f(y(r)) d r+\int_{0}^{t} S_{\lambda}(t-r) h(y(r)) d \theta_{\tau} \omega(r)
$$

and the right-hand side of the last equality is equal to $\varphi\left(t, \theta_{\tau} \omega, \varphi(\tau, \omega, x)\right)$.

The measurability of the mapping $\varphi$ follows due to its continuity with respect to $\omega$ (that implies measurability with respect to $\omega$ ), also due to its continuity with respect to $(t, x)$ for a fixed $\omega$ and the separability of $\ell^{2}$; see Lemma III.14 in Castaing and Valadier [10].

4. Exponential stability of the trivial solution. The purpose of this section is to show that the trivial solution of (3.1) is exponential stable. Therefore, we start assuming that zero is an equilibrium of the SLDS.

Since we work directly with our SLDS without transforming it into a random equation, the norm of the solution depends on how large the norm of the noisy input is, and therefore we will consider a cut-off strategy, in such a way that we will deal with a modified lattice system depending on a random variable. Further, that random variable can be chosen in a suitable way such that it turns out that it is possible to apply a Gronwall-like lemma, which together with the temperedness of the involved random variables will imply that the solution of the modified system coincides with that of the original lattice system, that converges to the trivial solution exponentially fast.

DEFINITION 4.1. The trivial solution of the SLDS is said to be exponential stable with rate $\mu>0$ if for almost every $\omega$ there exists a random variable $\alpha(\omega)>0$ and a random neighborhood $U(\omega)$ of zero such that for all $\omega \in \Omega$ and $t \in R^{+}$

$$
\sup _{x \in U(\omega)}\|\varphi(t, \omega, x)\| \leq \alpha(\omega) e^{-\mu t},
$$

where $\varphi: R^{+} \times \Omega \times \ell^{2} \rightarrow \ell^{2}$ is the cocycle mapping given in Theorem 3.7.

For the study of exponential stability of systems driven by continuous semimartingales, see the monograph [34]. In the spirit of working with the rich theory of RDS, here we have adapted the definition of exponential stability to the RDS setting.

We would like to prove that the trivial solution of the SLDS is exponentially stable with rate $\mu<\lambda$. In order to do that, first of all we need to introduce the key concept of temperedness. A random variable $R \in(0, \infty)$ is called tempered from above with respect to the metric dynamical system $(\Omega, \mathcal{F}, P, \theta)$ if

$$
\limsup _{t \rightarrow \pm \infty} \frac{\log ^{+} R\left(\theta_{t} \omega\right)}{t}=0 \quad \text { with probability } 1 \text {. }
$$

Therefore, temperedness from above describes the subexponential growth of a stochastic stationary process $(t, \omega) \mapsto R\left(\theta_{t} \omega\right)$. $R$ is called tempered from below if $R^{-1}$ is tempered from above. In particular, if the random variable $R$ is tempered from below and $t \mapsto R\left(\theta_{t} \omega\right)$ is continuous, then for any $\epsilon>0$ there exists a random variable $C_{\epsilon}(\omega)>0$ such that

$$
R\left(\theta_{t} \omega\right) \geq C_{\epsilon}(\omega) e^{-\epsilon|t|} \quad \text { with probability } 1 .
$$

A sufficient condition for temperedness with respect to an ergodic metric dynamical system is that

$$
E \sup _{t \in[0,1]} \log ^{+} R\left(\theta_{t} \omega\right)<\infty
$$


see Arnold [1, p. 165]. Hence, by Kunita [32, Theorem 1.4.1] we obtain that $R(\omega)=$ $\|\omega\|_{\beta^{\prime}, 0,1}$ is tempered from above because $\log ^{+} r \leq r$ for $r>0$ and trivially $\sup _{t \in[0,1]}$ $\left\|\theta_{t} \omega\right\|_{\beta, 0,1} \leq\|\omega\|_{\beta, 0,2}$. Furthermore, the set of all $\omega$ satisfying (4.1) is invariant with respect to the flow $\theta$.

We now introduce two more assumptions, which in particular imply that (3.1) has the unique trivial solution.

In what follows, for $\delta>0$ we also make these assumptions:

$\left(\mathrm{A} 3^{\prime}\right)$ Each $f_{i}$ is defined on $[-\delta, \delta]$. In addition to the assumption (A3), we assume that $\left.f_{i}(0)=f_{i}^{\prime}(0)=0, f_{i} \in C^{2}([-\delta, \delta]), R\right)$ and there exists a positive constant $M_{f}$ such that

$$
\left|f_{i}^{\prime \prime}(\zeta)\right| \leq M_{f}, \quad \zeta \in[-\delta, \delta], i \in Z .
$$

(A4 $\left.4^{\prime}\right)$ Let each $h_{i}$ be defined on $[-\delta, \delta]$. In addition to the assumption (A4), we assume that $h_{i}(0)=h_{i}^{\prime}(0)=0$.

The operators $f, h$ then are defined on $\bar{B}_{\ell^{2}}(0, \delta)$. In particular, from $\left(\mathrm{A} 3^{\prime}\right)$ we derive that $f$ is Fréchet differentiable and its derivative $D f: \ell^{2} \mapsto L\left(\ell^{2}\right)$ is continuous. Indeed, for $u, v \in \ell^{2}$ we obtain

$$
\|f(u+v)-f(u)-D f(u) v\|^{2} \leq \frac{1}{4} M_{f}^{2}\|v\|^{4}
$$

and

$$
\|D f(u)-D f(v)\|_{L\left(\ell^{2}\right)}^{2}=\sup _{\|z\|=1}\|D f(u) z-D f(v) z\|^{2} \leq M_{f}^{2}\|u-v\|^{2} .
$$

Furthermore, these assumptions ensure that (3.1) has the unique trivial solution.

We introduce $\chi$ to be the cut-off function

$$
\chi: \ell^{2} \rightarrow \bar{B}_{\ell^{2}}(0,1), \quad \chi(u)=\left\{\begin{array}{ccc}
u & : & \|u\| \leq \frac{1}{2} \\
0 & : & \|u\| \geq 1
\end{array}\right.
$$

such that the norm of $\chi(u)$ is bounded by 1 . We also assume that $\chi$ is twice continuously differentiable with bounded derivatives $D \chi$ and $D^{2} \chi$. Bounds of these derivatives are denoted by $L_{D \chi}, L_{D^{2} \chi}$. Now for $u \in \ell^{2}$ and some $0<\hat{R} \leq \delta$ we define

$$
\chi_{\hat{R}}(u)=\hat{R} \chi(u / \hat{R}) \in \bar{B}_{\ell^{2}}(0, \hat{R}) .
$$

Then it is easy to see that the first derivative $D \chi_{\hat{R}}$ of $\chi_{\hat{R}}$ is bounded by $L_{D \chi}$, while the second derivative $D^{2} \chi_{\hat{R}}$ is bounded by $\frac{L_{D^{2} \chi}}{\hat{R}}$.

We now modify the operators $f, h$ by considering their compositions with the above cut-off function. In that way, we set $f_{\hat{R}}:=f \circ \chi_{\hat{R}}: \ell^{2} \rightarrow \ell^{2}$ and $h_{\hat{R}}:=h \circ \chi_{\hat{R}}$ : $\ell^{2} \rightarrow L_{2}\left(\ell^{2}\right)$, consider (3.10) replacing $f$ by $f_{\hat{R}}$ and $h$ by $h_{\hat{R}}$, and the sequence $\left(u^{n}\right)_{n \in N}$ defined by

$$
\begin{aligned}
u^{n}(t)= & S_{\lambda}(t) u^{n}(0)+\int_{0}^{t} S_{\lambda}(t-r) f_{\hat{R}\left(\theta_{n} \omega\right)}\left(u^{n}(r)\right) d r \\
& +\int_{0}^{t} S_{\lambda}(t-r) h_{\hat{R}\left(\theta_{n} \omega\right)}\left(u^{n}(r)\right) d \theta_{n} \omega, \quad t \in[0,1],
\end{aligned}
$$

where $u^{0}(0)=x$ and $u^{n}(0)=u^{n-1}(1)$. Since the modified coefficients satisfy the assumptions in Theorem 3.4 for any $n \in N$, then there exists a unique solution $u^{n}$ to $(4.2)$ on $[0,1]$.

Next we establish a result which will be one of the keys in order to obtain the exponential stability of the trivial solution. 
Lemma 4.2. For every $R>0$ there exists a positive $\hat{R} \leq \delta$ such that for all $u, z \in \ell^{2}$

$$
\begin{aligned}
\left\|f_{\hat{R}}(u)\right\| & \leq R L_{D \chi}\|u\|, \\
\left\|h_{\hat{R}}(u)\right\|_{L_{2}\left(\ell^{2}\right)} & \leq R L_{D \chi}\|u\|, \\
\left\|h_{\hat{R}}(u)-h_{\hat{R}}(z)\right\|_{L_{2}\left(\ell^{2}\right)} & \leq R L_{D \chi}\|u-z\| .
\end{aligned}
$$

Proof. By $D f(0)=0$ and the continuity of $D f$, for any $R>0$ we can choose an $\hat{R} \leq \delta$ such that

$$
\sup _{\|v\| \leq \hat{R}}\|D f(v)\|_{L\left(\ell^{2}\right)} \leq R .
$$

Then for $u \in \ell^{2}$, since $f(0)=0$ from the mean value theorem we have

$$
\begin{aligned}
\left\|f_{\hat{R}}(u)\right\| \leq \sup _{z \in \ell^{2}}\left\|D\left(f\left(\chi_{\hat{R}}(z)\right)\right)\right\|_{L\left(\ell^{2}\right)}\|u\| & \leq \sup _{\|v\| \leq \hat{R}}\|D f(v)\|_{L\left(\ell^{2}\right)} \sup _{z \in \ell^{2}}\left\|D \chi_{\hat{R}}(z)\right\|_{L\left(\ell^{2}\right)}\|u\| \\
& \leq R L_{D \chi}\|u\|,
\end{aligned}
$$

and therefore (4.3) is shown. Following the same steps we prove (4.4).

Finally, by the regularity of $D h$,

$$
\begin{aligned}
\left\|h_{\hat{R}}(u)-h_{\hat{R}}(z)\right\|_{L_{2}\left(\ell^{2}\right)} & \leq \sup _{\|v\| \leq \hat{R}}\|D h(v)\|_{L\left(\ell^{2}, L_{2}\left(\ell^{2}\right)\right)}\left\|\chi_{\hat{R}}(u)-\chi_{\hat{R}}(z)\right\| \\
& \leq L_{D \chi} \sup _{\|v\| \leq \hat{R}}\|D h(v)\|_{L\left(\ell^{2}, L_{2}\left(\ell^{2}\right)\right)}\|u-z\| \leq R L_{D \chi}\|u-z\| .
\end{aligned}
$$

For $n \in Z^{+}$, we set

$$
u(t)=u^{n}(t-n) \quad \text { if } t \in[n, n+1] .
$$

Let us emphasize that the previous function $u$ is defined on the whole positive real line and is Hölder continuous on any interval $[n, n+1]$. However, we cannot claim yet that $u$ defined by (4.6) is our mild solution obtained in Theorem 3.4. The reason is that any $u^{n}$ is a solution of a modified lattice problem depending on the cut-off function $\chi_{\hat{R}}$ and driven by a path $\theta_{n} \omega$. But as we will show below, using the additivity of the integrals, the estimates of the functions $f_{\hat{R}}$ and $h_{\hat{R}}$ given in Lemma 4.2, and a suitable choice of the random variables $R$ and $\hat{R}$, we will end up proving that not only $u$ given by (4.6) is the solution of our original stochastic lattice system (3.10), but also that it converges to the trivial solution exponential fast with a certain decay rate $\mu$.

In order to prove the previous assertions, we first express $u$ given by (4.6) for $t \in[n, n+1]$ as follows:

$$
\begin{aligned}
& u(t)=S_{\lambda}(t-n) u(n)+\int_{n}^{t} S_{\lambda}(t-r) f_{\hat{R}\left(\theta_{n} \omega\right)}(u(r)) d r+\int_{n}^{t} S_{\lambda}(t-r) h_{\hat{R}\left(\theta_{n} \omega\right)}(u(r)) d \omega(r) \\
&=S_{\lambda}(t) x+ \sum_{j=0}^{n-1} S_{\lambda}(t-j-1)\left(\int_{j}^{j+1} S_{\lambda}(j+1-r) f_{\hat{R}\left(\theta_{j} \omega\right)}(u(r)) d r\right. \\
&\left.+\int_{j}^{j+1} S_{\lambda}(j+1-r) h_{\hat{R}\left(\theta_{j} \omega\right)}(u(r)) d \omega(r)\right)
\end{aligned}
$$

Copyright $@$ by SIAM. Unauthorized reproduction of this article is prohibited. 


$$
\begin{gathered}
\quad+\int_{n}^{t} S_{\lambda}(t-r) f_{\hat{R}\left(\theta_{n} \omega\right)}(u(r)) d r+\int_{n}^{t} S_{\lambda}(t-r) h_{\hat{R}\left(\theta_{n} \omega\right)}(u(r)) d \omega(r) \\
=S_{\lambda}(t) x+\sum_{j=0}^{n-1} S_{\lambda}(t-j-1)\left(\int_{0}^{1} S_{\lambda}(1-r) f_{\hat{R}\left(\theta_{j} \omega\right)}\left(u^{j}(r)\right) d r\right. \\
\left.\quad+\int_{0}^{1} S_{\lambda}(1-r) h_{\hat{R}\left(\theta_{j} \omega\right)}\left(u^{j}(r)\right) d \theta_{j} \omega(r)\right) \\
+\int_{0}^{t-n} S_{\lambda}(t-n-r) f_{\hat{R}\left(\theta_{n} \omega\right)}\left(u^{n}(r)\right) d r+\int_{0}^{t-n} S_{\lambda}(t-n-r) h_{\hat{R}\left(\theta_{n} \omega\right)}\left(u^{n}(r)\right) d \theta_{n} \omega(r),
\end{gathered}
$$

where this splitting is a consequence of the additivity of the integrals, Theorem 2.4 and (2.1).

Notice that in all the integrals on the right-hand side of the previous expression, the time varies in the interval $[0,1]$ (in the last two integrals, $[0, t-n]$ is contained in $[0,1])$. Hence, we are going to estimate the Hölder-norm of all these terms setting now $T_{1}=0$ and $T_{2}=1$. Due to the presence of the semigroup $S_{\lambda}$ as a factor in all terms under the sum, in the following estimates we do not need to consider the $\beta, \rho$-norm but the $\beta$-norm, that is, in what follows $\rho=0$.

Note that by (4.3) we have

$$
\left\|\int_{0} S_{\lambda}(\cdot-r) f_{\hat{R}\left(\theta_{n} \omega\right)}\left(u^{n}(r)\right) d r\right\|_{\infty} \leq R\left(\theta_{n} \omega\right) L_{D \chi}\left\|u^{n}\right\|_{\infty} .
$$

For the Hölder-seminorm, thanks to (3.4),

$$
\begin{aligned}
& \left\|\int_{0} S_{\lambda}(\cdot-r) f_{\hat{R}\left(\theta_{n} \omega\right)}\left(u^{n}(r)\right) d r\right\| \|_{\beta} \\
& =\sup _{0 \leq s<t \leq 1} \frac{\left\|\int_{s}^{t} S_{\lambda}(t-r) f_{\hat{R}\left(\theta_{n} \omega\right)}\left(u^{n}(r)\right) d r+\int_{0}^{s}\left(S_{\lambda}(t-r)-S_{\lambda}(s-r)\right) f_{\hat{R}\left(\theta_{n} \omega\right)}\left(u^{n}(r)\right) d r\right\|}{(t-s)^{\beta}} \\
& \leq \sup _{0 \leq s<t \leq 1}\left((t-s)^{1-\beta} \sup _{r \in[s, t]}\left(\left\|S_{\lambda}(t-r)\right\|_{L\left(\ell^{2}\right)}\left\|f_{\hat{R}\left(\theta_{n} \omega\right)}\left(u^{n}(r)\right)\right\|\right)\right) \\
& +\sup _{0 \leq s<t \leq 1}\left(\frac{s}{(t-s)^{\beta}} \sup _{r \in[0, s]}\left(\left\|S_{\lambda}(t-r)-S_{\lambda}(s-r)\right\|_{L\left(\ell^{2}\right)}\left\|f_{\hat{R}\left(\theta_{n} \omega\right)}\left(u^{n}(r)\right)\right\|\right)\right) \\
& \leq R\left(\theta_{n} \omega\right) L_{D \chi}\left\|u^{n}\right\|_{\infty}+\left\|A_{\lambda}\right\| R\left(\theta_{n} \omega\right) L_{D \chi}\left\|u^{n}\right\|_{\infty} .
\end{aligned}
$$

Then

$$
\left\|\int_{0} S_{\lambda}(\cdot-r) f_{\hat{R}\left(\theta_{n} \omega\right)}\left(u^{n}(r)\right) d r\right\|_{\beta} \leq\left(2+\left\|A_{\lambda}\right\|\right) R\left(\theta_{n} \omega\right) L_{D \chi}\left\|u^{n}\right\|_{\beta} .
$$

On the other hand, since $h(0)=0$, by $(4.4)$ and (4.5) we get

$$
\begin{aligned}
\left\|h_{\hat{R}\left(\theta_{n} \omega\right)}(u(\cdot))\right\|_{\beta} & =\sup _{t \in[0,1]}\left\|h_{\hat{R}\left(\theta_{n} \omega\right)}(u(t))\right\|_{L_{2}\left(\ell^{2}\right)} \\
& +\sup _{0 \leq r<q \leq 1} \frac{\left\|h_{\hat{R}\left(\theta_{n} \omega\right)}(u(r))-h_{\hat{R}\left(\theta_{n} \omega\right)}(u(q))\right\|_{L_{2}\left(\ell^{2}\right)}}{(r-q)^{\beta}} \\
& \leq L_{D \chi} R\left(\theta_{n} \omega\right)\|u\|_{\beta} .
\end{aligned}
$$

Copyright $\odot$ by SIAM. Unauthorized reproduction of this article is prohibited. 
For the stochastic integral, thanks to (3.12) and (3.13) we obtain

$$
\begin{aligned}
& \left\|\int_{0} S_{\lambda}(\cdot-r) h_{\hat{R}\left(\theta_{n} \omega\right)}\left(u^{n}(r)\right) d \theta_{n} \omega(r)\right\|_{\beta} \\
& \quad \leq c_{\beta, \beta^{\prime}}\left\|\theta_{n} \omega\right\|_{\beta^{\prime}}\left(1+\left\|A_{\lambda}\right\|\right)\left(2+\left\|A_{\lambda}\right\|\right)\left\|h_{\hat{R}\left(\theta_{n} \omega\right)}\left(u^{n}(\cdot)\right)\right\|_{\beta} \\
& \quad \leq L_{D \chi} c_{\beta, \beta^{\prime}}\left\|\theta_{n} \omega\right\|_{\beta^{\prime}}\left(1+\left\|A_{\lambda}\right\|\right)\left(2+\left\|A_{\lambda}\right\|\right) R\left(\theta_{n} \omega\right)\left\|u^{n}\right\|_{\beta},
\end{aligned}
$$

where $c_{\beta, \beta^{\prime}}$ denotes a constant that only depends on $\beta$ and $\beta^{\prime}$.

For the terms under the sum we have

$$
\begin{aligned}
& \left\|S_{\lambda}(\cdot-j-1) \int_{0}^{1} S_{\lambda}(1-r) f_{\hat{R}\left(\theta_{j} \omega\right)}\left(u^{j}(r)\right) d r\right\|_{\beta, n, n+1} \\
& \quad=\left\|S_{\lambda}(\cdot-j-1)\right\|_{\beta, n, n+1}\left\|\int_{0}^{1} S_{\lambda}(1-r) f_{\hat{R}\left(\theta_{j} \omega\right)}\left(u^{j}(r)\right) d r\right\|^{\cdot} \\
& \quad \leq\left\|S_{\lambda}(\cdot-j-1)\right\|_{\beta, n, n+1}\left\|\int_{0} S_{\lambda}(\cdot-r) f_{\hat{R}\left(\theta_{j} \omega\right)}\left(u^{j}(r)\right) d r\right\|_{\infty},
\end{aligned}
$$

and from Lemma 3.1,

$$
\left\|S_{\lambda}(\cdot-j-1)\right\|_{\beta, n, n+1} \leq\left(1+\left\|A_{\lambda}\right\|\right) e^{-\lambda(n-j-1)},
$$

so that

$$
\begin{gathered}
\left\|S_{\lambda}(\cdot-j-1) \int_{0}^{1} S_{\lambda}(1-r) f_{\hat{R}\left(\theta_{j} \omega\right)}\left(u^{j}(r)\right) d r\right\|_{\beta, n, n+1} \\
\quad \leq\left(1+\left\|A_{\lambda}\right\|\right) e^{-\lambda(n-j-1)} R\left(\theta_{j} \omega\right) L_{D \chi}\left\|u^{j}\right\|_{\beta} .
\end{gathered}
$$

Following similar steps, thanks to (3.12) we have

$$
\begin{aligned}
& \left\|S_{\lambda}(\cdot-j-1) \int_{0}^{1} S_{\lambda}(1-r) h_{\hat{R}\left(\theta_{j} \omega\right)}\left(u^{j}(r)\right) d \theta_{j} \omega(r)\right\|_{\beta, n, n+1} \\
& \quad \leq L_{D \chi} c_{\beta, \beta^{\prime}}\left\|\theta_{j} \omega\right\|_{\beta^{\prime}}\left(1+\left\|A_{\lambda}\right\|\right)^{2} e^{-\lambda(n-j-1)} R\left(\theta_{j} \omega\right)\left\|u^{j}\right\|_{\beta} .
\end{aligned}
$$

Therefore, taking the $\|\cdot\|_{\beta, n, n+1}$ norm of the different terms in (4.7), applying the triangle inequality, and in view of the above estimates, we obtain

$$
\begin{aligned}
\left\|u^{n}\right\|_{\beta} & \leq\left\|S_{\lambda}\right\|_{\beta, n, n+1}\|x\|+C \sum_{j=0}^{n-1} R\left(\theta_{j} \omega\right)\left(1+\left\|\theta_{j} \omega\right\|_{\beta^{\prime}}\right)\left\|u^{j}\right\|_{\beta} e^{-\lambda(n-j-1)} \\
& +C R\left(\theta_{n} \omega\right)\left(1+\left\|\theta_{n} \omega\right\|_{\beta^{\prime}}\right)\left\|u^{n}\right\|_{\beta}
\end{aligned}
$$

where $C=\max \left\{1, c_{\beta, \beta^{\prime}}\right\} L_{D \chi}\left(1+\left\|A_{\lambda}\right\|\right)\left(2+\left\|A_{\lambda}\right\|\right)$.

Let now $\epsilon \in(0,1)$, which will be determined later more precisely. Define the variables $R$ and $\hat{R}$ as follows:

$$
R(\omega)=\frac{\epsilon}{2 C\left(1+\|\omega\|_{\beta^{\prime}}\right)}
$$

and

$$
\begin{gathered}
\hat{R}(\omega)=\sup \left\{\hat{r} \in[0, \delta]:\|D f(v)\|_{L\left(\ell^{2}\right)}+\|D h(v)\|_{L\left(\ell^{2}, L_{2}\left(\ell^{2}\right)\right)} \leq R(\omega),\right. \\
\text { for all } v \in \bar{B}(0, \hat{r})\}
\end{gathered}
$$

Copyright $@$ ( ) by SIAM. Unauthorized reproduction of this article is prohibited. 
$\hat{R}(\omega)$ is a random variable; see [20]. In addition, since $\|\omega\|_{\beta^{\prime}}$ is tempered from above then $R$ is tempered from below. According to Lemma A.3 it follows that $\hat{R}$ is tempered from below. In the contrary case we have $\omega \in \Omega, \mu \in R^{+} \backslash\{0\} \cup\{+\infty\}$ and a sequence $\left(t_{i}\right)_{i \in N}$ tending to $+\infty$ or $-\infty$ such that

$$
\hat{R}\left(\theta_{t_{i}} \omega\right) \leq e^{-\mu\left|t_{i}\right|} .
$$

But then for sufficiently large $i$ we have

$$
R\left(\theta_{t_{i}} \omega\right) \leq \kappa e^{-\mu\left|t_{i}\right|},
$$

contradicting the temperedness of $R$ (here we have applied Lemma A.3, with $V=\ell^{2}$, $W=L\left(\ell^{2}\right) \times L\left(\ell^{2}, L_{2}\left(\ell^{2}\right)\right)$ and the function $F(z)=(D f(z), D h(z))$. Notice that the assumptions of that lemma are fulfilled thanks to the regularity properties of the functions $f$ and $h$. In particular, we need any $f_{i}$ to be two times differentiable.

With the above choice of $R$, coming back to (4.8), since $\epsilon<1$ we obtain

$$
\frac{1}{2}\left\|u^{n}\right\|_{\beta} \leq\left\|S_{\lambda}\right\|_{\beta, n, n+1}\|x\|+\frac{\epsilon}{2} \sum_{j=0}^{n-1} e^{-\lambda(n-j-1)}\left\|u^{j}\right\|_{\beta},
$$

hence

$$
\left\|u^{n}\right\|_{\beta} \leq 2\left(1+\left\|A_{\lambda}\right\|\right)\|x\| e^{-\lambda n}+\epsilon \sum_{j=0}^{n-1} e^{-\lambda(n-j-1)}\left\|u^{j}\right\|_{\beta} .
$$

Defining $y_{n}=\left\|u^{n}\right\|_{\beta} e^{\lambda n}, c=2\left(1+\left\|A_{\lambda}\right\|\right)$, and $g_{j}=\epsilon e^{\lambda}$, Lemma A.1 ensures that

$$
y_{n} \leq 2\left(1+\left\|A_{\lambda}\right\|\right)\|x\| \prod_{i=0}^{n-1}\left(1+\epsilon e^{\lambda}\right)=2\left(1+\left\|A_{\lambda}\right\|\right)\|x\|\left(1+\epsilon e^{\lambda}\right)^{n},
$$

hence

$$
\left\|u^{n}\right\|_{\beta} \leq 2\left(1+\left\|A_{\lambda}\right\|\right)\|x\| e^{-n\left(\lambda-\log \left(1+\epsilon e^{\lambda}\right)\right)} .
$$

On the other hand, due to Lemma A.2, since $\hat{R}(\omega) / 2$ is tempered from below, we can find a zero neighborhood $U$ depending on $\omega$ such that for $x$ contained in this neighborhood we have

$$
\left\|u^{n}\right\|_{\beta} \leq \frac{\hat{R}\left(\theta_{n} \omega\right)}{2} \text { for all } n \in Z^{+},
$$

As we will show in the next result, (4.10) is a crucial estimate to prove that the sequence of truncated solutions $\left(u^{n}\right)_{n \in N}$ defines a solution of (3.10) on $R^{+}$. In fact, thanks to the previous considerations, we can state the main result of the paper.

THEOREM 4.3. Suppose that conditions (A1) $-(\mathrm{A} 4)$ and $\left(\mathrm{A} 3^{\prime}\right)-\left(\mathrm{A} 4^{\prime}\right)$ hold and consider $\epsilon(\lambda)=\epsilon \in\left(0,1-e^{-\lambda}\right)$. Then the trivial solution is exponentially stable with an exponential rate less than or equal to $\mu<\lambda-\log \left(1+\epsilon e^{\lambda}\right)$.

Proof. First of all, let us prove that $u$ given by (4.6) is a solution of our original stochastic lattice system. In fact, from (4.10) we deduce that for any $j \in Z^{+}$and any $r \in[0,1]$ we have that

$$
\left\|u^{j}(r)\right\| \leq \frac{\hat{R}\left(\theta_{j} \omega\right)}{2}
$$

Copyright $@$ by SIAM. Unauthorized reproduction of this article is prohibited. 
Consequently $\chi_{\hat{R}\left(\theta_{j} \omega\right)}\left(u^{j}(r)\right)=u^{j}(r)$, hence

$$
f_{\hat{R}\left(\theta_{j} \omega\right)}\left(u^{j}(r)\right)=f\left(u^{j}(r)\right), \quad h_{\hat{R}\left(\theta_{j} \omega\right)}\left(u^{j}(r)\right)=h\left(u^{j}(r)\right)
$$

for any $r \in[0,1]$ and $j \in Z^{+}$. Then $u$ given by (4.6), where $u^{n}$ solves (4.2), is a solution of (3.10) on $R^{+}$.

Now we show the exponential stability of the trivial solution, according to Definition 4.1. Take $\epsilon \in\left(0,1-e^{-\lambda}\right), \hat{\epsilon}>0$, small enough and $\mu<\lambda-\log \left(1+\epsilon e^{\lambda}\right)-\hat{\epsilon}$. From (4.9) we derive that there exist $T_{0}(\omega, \epsilon) \in N$ and a neighborhood of zero $U(\omega)$ such that if $x \in U(\omega)$, for $t \geq T_{0}(\omega, \epsilon)$

$$
\|\varphi(t, \omega, x)\| \leq \alpha_{1}(\omega) e^{-\left(\lambda-\log \left(1+\epsilon e^{\lambda}\right)-\hat{\epsilon}\right) t} \leq \alpha_{1}(\omega) e^{-\mu t},
$$

where the positive random variable $\alpha_{1}(\omega)$ depends on the coefficients $\kappa$ and $\left\|A_{\lambda}\right\|$.

On the other hand, from (4.10) we derive

$$
\sup _{x \in U(\omega), t \in\left[0, T_{0}(\omega, \epsilon)\right]}\|\varphi(t, \omega, x)\| \leq \sup _{x \in U(\omega), 0 \leq n \leq T_{0}(\omega, \epsilon)}\left\|u^{n}\right\|_{\beta} \leq \alpha_{2}(\omega) e^{-\mu t},
$$

where

$$
\alpha_{2}(\omega)=\sup _{0 \leq n \leq T_{0}(\omega, \epsilon)} \frac{\hat{R}\left(\theta_{n} \omega\right)}{2} e^{\mu T_{0}}
$$

Therefore,

$$
\begin{aligned}
\sup _{x \in U(\omega)}\|\varphi(t, \omega, x)\| & \leq \sup _{x \in U(\omega), t \in\left[0, T_{0}(\omega, \epsilon)\right]}\|\varphi(t, \omega, x)\|+\sup _{x \in U(\omega), t \geq T_{0}(\omega, \epsilon)}\|\varphi(t, \omega, x)\| \\
& \leq\left(\alpha_{1}(\omega)+\alpha_{2}(\omega)\right) e^{-\mu t} .
\end{aligned}
$$

Appendix A. In this section we present some technical results that we have used in section 4 .

First of all, we introduce a discrete Gronwall-like lemma, whose proof can be derived easily from Lemma 100 in [17].

Lemma A.1. Let $\left(y_{n}\right)$ and $\left(g_{n}\right)$ be nonnegative sequences and $c$ a nonnegative constant. If

$$
y_{n} \leq c+\sum_{j=0}^{n-1} g_{j} y_{j},
$$

then

$$
y_{n} \leq c \prod_{j=0}^{n-1}\left(1+g_{j}\right) .
$$

Lemma A.2. Suppose that $R_{i} \geq C_{\epsilon} e^{-\epsilon i}$ for any $0<\epsilon<\sigma$ and $i \in N$, where $C_{\epsilon}>0$. Let $\left(v_{i}\right)_{i \in N}$ be a sequence such that $v_{i} \leq v_{0} e^{-\sigma i}$. Then for sufficiently small $v_{0}$ we have for any $i \in N$

$$
v_{i} \leq R_{i}
$$

The proof of this result follows easily. Note that if, for instance, we assume that a random variable $R>0$ is tempered from below, then we can find a random variable $C_{\epsilon}>0$ such that $v_{i}<R\left(\theta_{i} \omega\right)$ holds for $v_{0}<C_{\epsilon}(\omega)$.

The following result establishes the relationship between the random variables $R$ and $\hat{R}$ as needed in section 3 .

Copyright $@$ by SIAM. Unauthorized reproduction of this article is prohibited. 
Lemma A.3. Let $\left(V,\|\cdot\|_{V}\right)$ and $\left(W,\|\cdot\|_{W}\right)$ be some Banach spaces, $\delta>0$, and let $F \not \equiv 0$ be a function from $\bar{B}_{V}(0, \delta)$ into $W$ with $F(0)=0$ which is continuously differentiable such that

$$
\sup _{z \in \bar{B}_{V}(0, \delta)}\|D F(z)\|_{L(V, W)}=\kappa<\infty
$$

Consider the centered open balls $B_{W}(0, R), B_{V}(0, \hat{R})$, where $R>0$ and $\hat{R}=\hat{R}(R) \leq \delta$ is the supremum of all numbers $\hat{r}>0$ such that

$$
B_{V}(0, \hat{r}) \subset F^{-1}\left(B_{W}(0, R)\right) .
$$

Then, for $0 \leq R<\sup \left\{\|F(z)\|_{W}, z \in \bar{B}_{V}(0, \delta)\right\}$, we obtain

$$
\sup _{z \in \bar{B}_{V}(0, \hat{R}(R))}\|F(z)\|_{W} \leq R, \quad \frac{\hat{R}(R)}{R} \geq \frac{1}{\kappa} \in(0, \infty] .
$$

Proof. Denote

$$
f_{F}: \bar{B}_{V}(0, \delta) \rightarrow R^{+}, f_{F}(z)=\|F(z)\|_{W}, z \in \bar{B}_{V}(0, \delta) .
$$

Let us define

$$
\hat{R}=\sup \left\{\hat{r} \in[0, \delta]: B_{V}(0, \hat{r}) \cap f_{F}^{-1}(\{R\})=\emptyset\right\} .
$$

Note that $f_{F}^{-1}(\{R\}) \neq \emptyset$ since $R \in f_{F}\left(\bar{B}_{V}(0, \delta)\right)$. Moreover, the set defining $\hat{R}$ is nonempty since

$$
f_{F}^{-1}([0, R)) \cap f_{F}^{-1}(\{R\})=\emptyset, \quad 0 \in f_{F}^{-1}([0, R)) ;
$$

therefore by the continuity of $f_{F}$ there exists always a positive $\hat{r}$ such that $B_{V}(0, \hat{r}) \subset$ $f_{F}^{-1}([0, R))$.

On the other hand, the ball $B_{V}(0, \hat{R})$ does not contain a $\hat{z}$ such that $R<f_{F}(\hat{z})=$ : $R_{1}$. In the other case, by the continuity of $f_{F}$, the set $f_{F}\left(B_{V}(0,\|\hat{z}\|)\right)$ would contain the interval $\left[0, R_{1}\right)$ which includes $R$ and there would exist a $\tilde{z} \in V$ with

$$
\|\tilde{z}\| \leq\|\hat{z}\|<\hat{R}, \quad f_{F}(\tilde{z})=R,
$$

which contradicts the definition of $\hat{R}$. Note that by the connectedness of balls their images by a continuous function are intervals. Hence

$$
B_{V}(0, \hat{R}) \cap f_{F}^{-1}((R, \infty))=\emptyset \quad \text { and } \quad \bar{B}_{V}(0, \hat{R}) \cap f_{F}^{-1}((R, \infty))=\emptyset,
$$

which proves the first part of (A.1).

Furthermore, by the definition of $\hat{R}$ for every $\varepsilon>0$ sufficiently small there exist $x_{\varepsilon}^{R} \in B_{V}(0, \hat{R})$, with $\inf _{\varepsilon>0}\left\|x_{\varepsilon}^{R}\right\|_{V}>0$, and $y_{\varepsilon}^{R} \in f_{F}^{-1}(\{R\})$ such that $\left\|x_{\varepsilon}^{R}-y_{\varepsilon}^{R}\right\|_{V}<\varepsilon$. Furthermore, by Taylor's formula

$$
f_{F}\left(x_{\varepsilon}^{R}\right) \leq \sup _{z \in \bar{B}_{V}(0, \rho)}\|D F(z)\|_{L(V, W)}\left\|x_{\varepsilon}^{R}\right\|_{V},
$$

and hence, applying again Taylor's formula, we get

$$
\begin{aligned}
f_{F}\left(y_{\varepsilon}^{R}\right) & \leq f_{F}\left(x_{\varepsilon}^{R}\right)+\left\|F\left(x_{\varepsilon}^{R}\right)-F\left(y_{\varepsilon}^{R}\right)\right\|_{W} \leq f_{F}\left(x_{\varepsilon}^{R}\right)+\varepsilon \sup _{z \in \bar{B}_{V}(0, \delta)}\|D F(z)\|_{L(V, W)} \\
& \leq \sup _{z \in \bar{B}_{V}(0, \delta)}\|D F(z)\|_{L(V, W)}\left(\left\|x_{\varepsilon}^{R}\right\|_{V}+\varepsilon\right) \leq \kappa\left(\left\|x_{\varepsilon}^{R}\right\|_{V}+\varepsilon\right) .
\end{aligned}
$$

Copyright $@$ by SIAM. Unauthorized reproduction of this article is prohibited. 
Therefore,

$$
\frac{\hat{R}}{R}=\lim _{\varepsilon \rightarrow 0} \frac{\left\|x_{\varepsilon}^{R}\right\|_{V}}{f_{F}\left(y_{\varepsilon}^{R}\right)} \geq \frac{1}{\kappa} \lim _{\varepsilon \rightarrow 0} \frac{\left\|x_{\varepsilon}^{R}\right\|_{V}}{\left\|x_{\varepsilon}^{R}\right\|_{V}+\varepsilon}=\frac{1}{\kappa} .
$$

Note that it is not necessary to consider $F \equiv 0$, because the results derived from the last lemma follow trivially.

\section{REFERENCES}

[1] L. ARnold, Random Dynamical Systems, Springer-Verlag, Berlin, 1998.

[2] P. Bates, H. Lisei, AND K. Lu, Attractors for stochastic lattice dynamical systems, Stoch. Dyn., 6 (2006), pp. 1-21.

[3] P. Bates, K. Lu, And B. Wang, Attractors for lattice dynamical systems, Internat. J. Bifur. Chaos, 11 (2001), pp. 143-153.

[4] H. Bauer, Probability Theory, de Gruyter Stud. Math. 23, Walter de Gruyter, Berlin, 1996, https://doi.org/10.1515/9783110814668.

[5] J. BeLL, Some threshold results for models of myelinated nerves, Math. Biosci., 54 (1981), pp. 181-190.

[6] J. BELl AND C. CosNer, Threshold behaviour and propagation for nonlinear differentialdifference systems motivated by modeling myelinated axons, Quart. Appl. Math., 42 (1984), pp. $1-14$.

[7] H. Bessaih, M. Garrido-Atienza, and B. Schmalfuss, Stochastic shell models driven by a multiplicative fractional Brownian motion, Phys. D, 320 (2016), pp. 38-56.

[8] T. Caraballo, X. Han, B. Schmalfuss, and J. Valero, Random attractors for stochastic lattice dynamical systems with infinite multiplicative white noise, Nonlinear Anal., 130 (2016), pp. 255-278.

[9] T. Caraballo and K. Lu, Attractors for stochastic lattice dynamical systems with a multiplicative noise, Front. Math. China, 3 (2008), pp. 317-335.

[10] C. Castaing and M. Valadier, Convex Analysis and Measurable Multifunctions, Lecture Notes in Math., 580. Springer-Verlag, Berlin, 1977.

[11] Y. Chen, H. Gao, M. Garrido-Atienza, and B. Schmalfuss, Pathwise solutions of SPDEs driven by Hölder-continuous integrators with exponent larger than $1 / 2$ and random dynamical systems, Discrete Contin. Dyn. Syst., 34 (2014), pp. 79-98, https://doi.org/10. 3934/dcds.2014.34.79.

[12] S.-N. Chow and J. Mallet-Paret, Pattern formulation and spatial chaos in lattice dynamical systems: I, IEEE Trans. Circuits Syst., 42 (1995), pp. 746-751.

[13] S.-N. Chow, J. Mallet-Paret, and E. S. V. Vleck, Pattern formation and spatial chaos in spatially discrete evolution equations, Random Comput. Dynam., 4 (1996), pp. 109-178.

[14] L. O. Chua And T. Roska, The CNN paradigm, IEEE Trans. Circuits Syst., 40 (1993), pp. 147156.

[15] L. O. Chua And L. Yang, Cellular neural networks: Applications, IEEE Trans. Circuits Syst., 35 (1988), pp. 1273-1290.

[16] L. O. Chua And L. YAng, Cellular neural networks: Theory, IEEE Trans. Circuits Syst., 35 (1988), pp. $1257-1272$.

[17] S. S. Dragomir, Some Gronwall Type Inequalities and Applications, Nova Science Publishers, Happauge, NY, 2003.

[18] T. ERneux And G. Nicolis, Propagating waves in discrete bistable reaction diffusion systems, Phys. D, 67 (1993), pp. 237-244.

[19] M. Garrido-Atienza, H. D. Luu, A. Neuenkirch, and B. Schmalfuss, Global stability of stochastic evolution equations driven by fractional Brownian motion with Hurst parameter in $(1 / 2,1)$, submitted.

[20] M. Garrido-Atienza, A. Neuenkirch, and B. Schmalfuss, Asymptotical stability of differential equations driven by Hölder-continuous paths, J. Dynam. Differential Equations, doi:10.1007/s10884-017-9574-6.

[21] M. J. Garrido-Atienza, K. Lu, and B. Schmalfuss, Random dynamical systems for stochastic partial differential equations driven by a fractional Brownian motion, Discrete Contin. Dyn. Syst. Ser. B, 14 (2010), pp. 473-493.

[22] M. J. Garrido-Atienza, K. Lu, and B. Schmalfuss, Unstable invariant manifolds for stochastic PDEs driven by a fractional Brownian motion, J. Differential Equations, 248 (2010), pp. 1637-1667.

Copyright $@$ by SIAM. Unauthorized reproduction of this article is prohibited. 
[23] M. J. Garrido-Atienza, K. Lu, and B. Schmalfuss, Local pathwise solutions to stochastic evolution equations driven by fractional Brownian motions with Hurst parameters $h \in$ (1/3,1/2], Discrete Contin. Dyn. Syst. Ser. B, 20 (2015), pp. 2553-2581.

[24] M. J. Garrido-Atienza, K. Lu, and B. Schmalfuss, Random dynamical systems for stochastic evolution equations driven by multiplicative fractional Brownian noise with Hurst parameters $h \in(1 / 3,1 / 2]$, SIAM J. Appl. Dyn. Syst., 15 (2016), pp. 625-654.

[25] M. J. Garrido-Atienza, B. Maslowski, and B. Schmalfuss, Random attractors for stochastic equations driven by a fractional Brownian motion, Internat. J. Bifur. Chaos, 20 (2010), pp. $1-22$.

[26] W. Gerstner, W. M. Kistler, R. Naud, and L. Paninski, Neuronal Dynamics, Cambridge University Press, Cambridge, UK, 2014.

[27] X. HAN, Asymptotic dynamics of stochastic lattice systems: A review, in Continuous and Distributed Systems II, V. A. Sadovnichiy and M. Z. Zgurovsky, eds., Springer-Verlag, Berlin, 2015, pp. 121-137.

[28] X. Han, W. Shen, and S. Zhou, Random attractors for stochastic lattice dynamical systems in weighted spaces, J. Differential Equations, 250 (2011), pp. 1235-1266.

[29] R. KAPVAL, Discrete models for chemically reacting systems, J. Math. Chem., 6 (1991), pp. 113163.

[30] J. P. KeEner, Propagation and its failure in coupled systems of discrete excitable cells, SIAM J. Appl. Math., 47 (1987), pp. 556-572.

[31] J. P. KEENER, The effects of discrete gap junction coupling on propagation in myocardium, J. Theor. Biol., 148 (1991), pp. 49-82.

[32] H. Kunita, Stochastic Flows and Stochastic Differential Equations, Cambridge University Press, Cambridge, UK, 1990.

[33] J. P. Laplante And T. ERneux, Propagating failure in arrays of coupled bistable chemical reactors, J. Phys. Chem., 96 (1992), pp. 4931-4934.

[34] X. MaO, Exponential Stability of Stochastic Differential Equations, Monogr. Textb. Pure Appl. Math., 182, Marcel Dekker, New York, 1994.

[35] Y. S. Mishura, Stochastic Calculus for Fractional Brownian Motion and Related Processes, Lecture Notes in Math. 1929, Springer-Verlag, Berlin, 2008.

[36] D. Nualart and A. RĂşCAnu, Differential equations driven by fractional Brownian motion, Collect. Math., 53 (2002), pp. 55-81.

[37] A. Pérez-Muñuzuri, V. Pérez-Muñuzuri, V. Pérez-Villar, and L. O. Chua, Spiral waves on a 2-d array of nonlinear circuits, IEEE Trans. Circuits Syst., 40 (1993), pp. 872-877.

[38] N. Rashevsky, Mathematical Biophysics, Dover, New York, 1960.

[39] S. Samko, A. Kilbas, And O. Marichev, Fractional Integrals and Derivatives: Theory and Applications, Gordon and Breach, Philadelphia, 1993.

[40] A. Scott, Analysis of a myelinated nerve model, Bull. Math. Biophys., 26 (1964), pp. 247-254.

[41] M. YAmada AND K. OhKITANI, Lyapunov spectrum of a chaotic model of three-dimensional turbulence, J. Phys. Soc. Jpn., 56 (1987), p. 4210.

[42] M. Z̈̈HLE, Integration with respect to fractal functions and stochastic calculus. I, Probab. Theory Related Fields, 111 (1998), pp. 333-374.

Copyright (C) by SIAM. Unauthorized reproduction of this article is prohibited. 